\title{
Congenital pituitary hypoplasia model demonstrates hypothalamic OTX2 regulation of pituitary progenitor cells
}

\author{
Ryusaku Matsumoto, ${ }^{1,2,3}$ Hidetaka Suga, ${ }^{4}$ Takashi Aoi, ${ }^{2,3}$ Hironori Bando, ${ }^{1}$ Hidenori Fukuoka, ${ }^{5}$ Genzo Iguchi, ${ }^{5,6,7}$ Satoshi Narumi, \\ Tomonobu Hasegawa, ${ }^{9}$ Keiko Muguruma, ${ }^{10,11}$ Wataru Ogawa, ${ }^{1}$ and Yutaka Takahashi' \\ 'Division of Diabetes and Endocrinology, Department of Internal Medicine, and 2Department of iPS cell Applications, Kobe University Graduate School of Medicine, Kobe, Hyogo, Japan. ${ }^{3}$ Department of \\ Advanced Medical Science, Kobe University Graduate School of Science, Technology, and Innovation, Kobe, Hyogo, Japan. ${ }^{4}$ Department of Diabetes and Endocrinology, Nagoya University Hospital, Nagoya, \\ Aichi, Japan. ${ }^{5}$ Department of Diabetes and Endocrinology, Kobe University Hospital, Kobe, Hyogo, Japan. ${ }^{6}$ Medical Center for Student Health, Kobe University, Kobe, Hyogo, Japan. ${ }^{7}$ Department of Biosignal \\ Pathophysiology, Kobe University Graduate School of Medicine, Kobe, Hyogo, Japan. ${ }^{8}$ Department of Molecular Endocrinology, National Research Institute for Child Health and Development, Tokyo, Japan. \\ ${ }^{9}$ Department of Pediatrics, Keio University School of Medicine, Tokyo, Japan. ${ }^{10}$ Laboratory for Cell Asymmetry, RIKEN Center for Biosystems Dynamics Research, Kobe, Hyogo, Japan. "Department of iPS Cell \\ Applied Medicine, Graduate School of Medicine, Kansai Medical University, Hirakata, Osaka, Japan.
}

\begin{abstract}
Pituitary develops from oral ectoderm in contact with adjacent ventral hypothalamus. Impairment in this process results in congenital pituitary hypoplasia (CPH); however, there have been no human disease models for CPH thus far, prohibiting the elucidation of the underlying mechanisms. In this study, we established a disease model of CPH using patient-derived induced pluripotent stem cells (iPSCs) and 3D organoid technique, in which oral ectoderm and hypothalamus develop simultaneously. Interestingly, patient iPSCs with a heterozygous mutation in the orthodenticle homeobox 2 (OTX2) gene showed increased apoptosis in the pituitary progenitor cells, and the differentiation into pituitary hormone-producing cells was severely impaired. As an underlying mechanism, ОTX2 in hypothalamus, not in oral ectoderm, was essential for progenitor cell maintenance by regulating LHX3 expression in oral ectoderm via FGF10 expression in the hypothalamus. Convincingly, the phenotype was reversed by the correction of the mutation, and the haploinsufficiency of OTX2 in control iPSCs revealed a similar phenotype, demonstrating that this mutation was responsible. Thus, we established an iPSC-based congenital pituitary disease model, which recapitulated interaction between hypothalamus and oral ectoderm and demonstrated the essential role of hypothalamic OTX2.
\end{abstract}

\section{Introduction}

Anterior pituitary develops from the oral ectoderm in contact with the adjacent hypothalamus progenitor. Although interaction between hypothalamus and oral ectoderm is supposed to play a crucial role in this process, the underlying mechanisms are not fully understood. This process includes a complex cascade of transcription factors and signaling molecules regulated by various growth/ differentiation factors $(1,2)$, including bone morphogenetic protein 4 (BMP4), sonic hedgehog (SHH), fibroblast growth factors (FGFs) 8 and 10, and Wnt5a. Congenital hypopituitarism is a multifactorial disorder that is usually associated with pituitary hypoplasia (3). So far, genetic studies in individuals with congenital hypopituitarism have revealed pathogenic mutations mainly in the genes encoding transcription factors, including POU1F1, PROP1, HESX1, LHX3, LHX4, OTX2, GLI2, SOX2, and SOX3 (4). These mutations demonstrate solely pituitary hormonal defects or syndromic pituitary hypoplasia generally involved in midline structural abnormalities.

Conflict of interest: The authors have declared that no conflict of interest exists. Copyright: () 2020, American Society for Clinical Investigation.

Submitted: January 15, 2019; Accepted: October 15, 2019; Published: December 17, 2019. Reference information: / Clin Invest. 2020;130(2):641-654.

https://doi.org/10.1172/JCl127378.
Knockout mouse studies of these genes have demonstrated their roles in pituitary development; however, phenotypes of knockout mice were not necessarily identical to that of humans with deleterious mutations in the same gene $(5,6)$. Especially in humans, the precise underlying mechanisms remain largely unknown, owing to the lack of a human pituitary developmental model.

Orthodenticle homeobox 2 (OTX2) plays an important role in the development of forebrain, eye, and pituitary (7-9). OTX2 mutations cause congenital pituitary hypoplasia $(\mathrm{CPH})$ and hypopituitarism with a variable severity $(10,11)$. In addition, eye malformation, such as anophthalmia and microphthalmia, is often observed (12). It has been shown that OTX2 is expressed in both oral ectoderm and hypothalamus (13). OTX2 protein binds to the promoters of HESX1 and POU1F1, which are expressed in oral ectoderm and required for pituitary development, and directly upregulates their expression $(11,12,14)$, suggesting the role of OTX2 expressed in the oral ectoderm. On the other hand, it has been shown that not oral ectoderm-specific but hypothalamus-specific Otx2-deficient mice exhibited impaired anterior pituitary development, suggesting that the OTX2 expressed in the hypothalamus plays an important role in pituitary development $(15,16)$; however, the precise underlying mechanisms, especially in humans, remain unclear. 
A

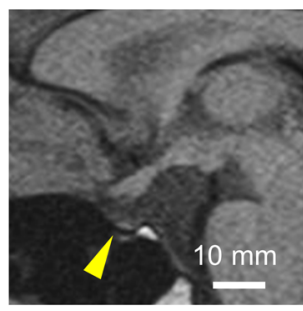

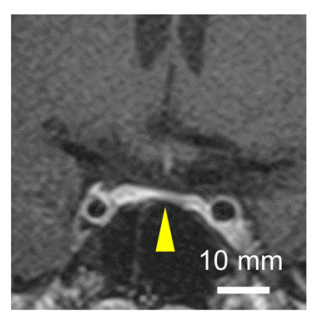

B
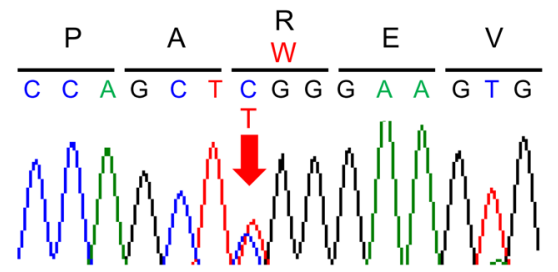

OTX2 R127W

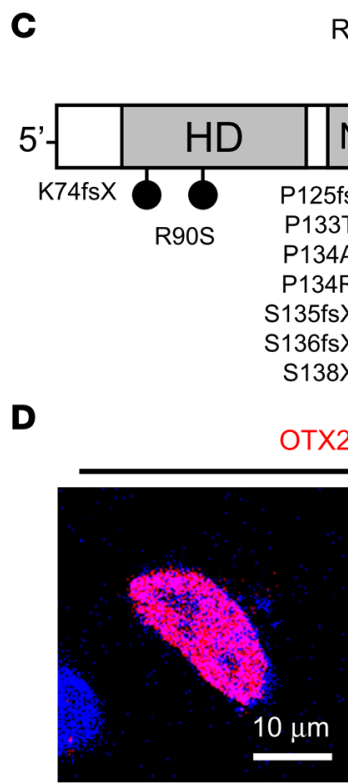

WT
R127W

P

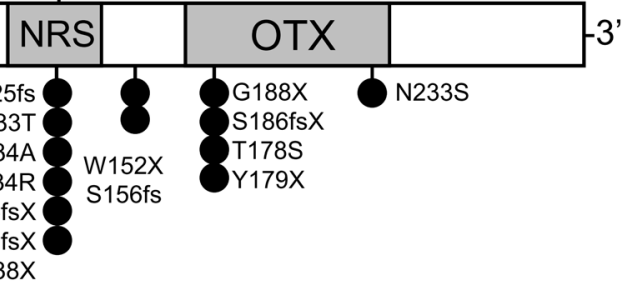

E

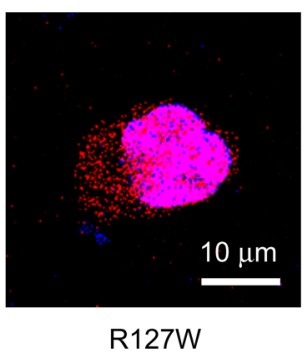

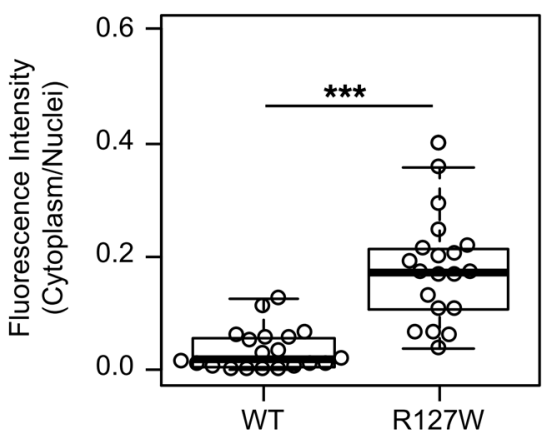

Figure 1. Patient characteristics and detection of a mutation in OTX2. (A) MRI of the patient. She showed severe pituitary hypoplasia. There were no signs of ectopic posterior pituitary and septo-optic dysplasia. (B) Sanger sequence revealed a heterozygous mutation in OTX2 (R127W). (C) The localization of the mutation in OTX2 and mutations previously reported in patients with $\mathrm{CPH}$. There is a hot spot in the nuclear retention signal (NRS) region. HD, homeodomain.

(D) Wild-type and mutant OTX2 were expressed in HEK293T cells. The mutant OTX2 exhibited an impaired nuclear localization. (E) Quantitative analysis of nuclear localization of wild-type and mutant OTX2. The line within the box indicates the median, the edge of the box represents the first and third quartiles, and the whiskers are the range of data excluding outliers; $n=20$ per group. ${ }^{* *} P<0.001$, Wilcoxon rank-sum test.
Human induced pluripotent stem cells (iPSCs) are promising tools for studying the process of human organ development and its disorders (17). Thus far, various organs have been induced from iPSCs in vitro, and disease models for genetic disorders have been successfully created (18). Recently, in vitro differentiation of anterior pituitary from pluripotent stem cells has been established. Studer et al. reported a 2-dimensional culture method in which functional anterior pituitary cells were induced with high efficiency in a short period $(19,20)$. Suga et al. reported a 3-dimensional method $(21,22)$. This method allowed induction of both the oral ectoderm and hypothalamus progenitor simultaneously and recapitulates the interaction between these tissues in vitro, which leads to anterior pituitary self-organization (23). This method is considered more suitable for analysis of the pathogenesis of the disease involved in the impairment of interaction between the hypothalamus and pituitary. Nevertheless, there have been no reports on disease models of human pituitary disorders. Here, we established a disease model of CPH using patient-derived iPSCs, which helped to reveal the role of hypothalamic OTX2 and the underlying mechanisms in pituitary development.

\section{Results}

Patient with CPH harbors OTX2 mutation. A 23-year-old woman was referred to our hospital for endocrinological examinations. She was born from healthy non-consanguineous parents at gestational age 41 weeks after an uneventful pregnancy and delivery. She did not show any apparent malformation in the eyes or brain. She exhibited growth retardation soon after birth, suggesting the presence of congenital growth hormone (GH) deficiency. At the age of 3 years, she was diagnosed with pan-hypopituitarism associated with pituitary hypoplasia. She was treated with pituitary hormone replacement therapy, including hydrocortisone, thyroxine, $\mathrm{GH}$, and Kaufmann's treatment. MRI of pituitary exhibited a severely hypomorphic pituitary (Figure 1A). The provocative test for anterior pituitary hormones revealed the presence of pan-hypopituitarism (Supplemental Table 1; supplemental material available online with this article; https://doi.org/10.1172/JCI127378DS1). Exome sequencing analysis of genomic DNA revealed a heterozygous variant (p.R127W; NCBI NP_068374) in OTX2 (Figure 1B), and no other mutations were found in the known genes associated with pituitary hypoplasia. The variant was present in none of the mutation/polymorphism databases we searched, including the Human Gene Mutation Database, the Genome Aggregation Database (gnomAD), 1000 Genomes Projects, and dbSNP147 (NCBI). The Arg127 residue is evolutionarily conserved among the vertebrates. This variant was located in the nuclear retention signal (7), which is a hot spot for the mutations (refs. 10, 24, and Figure $1 C)$, suggesting that this mutation is responsible for the disease and nuclear translocation of the mutant OTX2 may be impaired. Indeed, in contrast to wild-type OTX2, which localized in the nucleus, R127W-OTX2 showed impairment of nuclear translocation that is essential for the function of transcription factors 
A

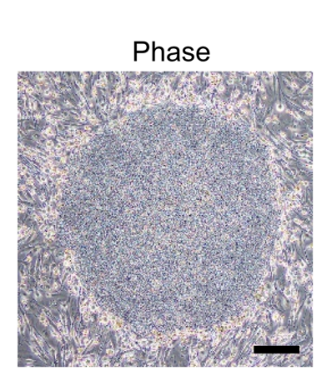

B

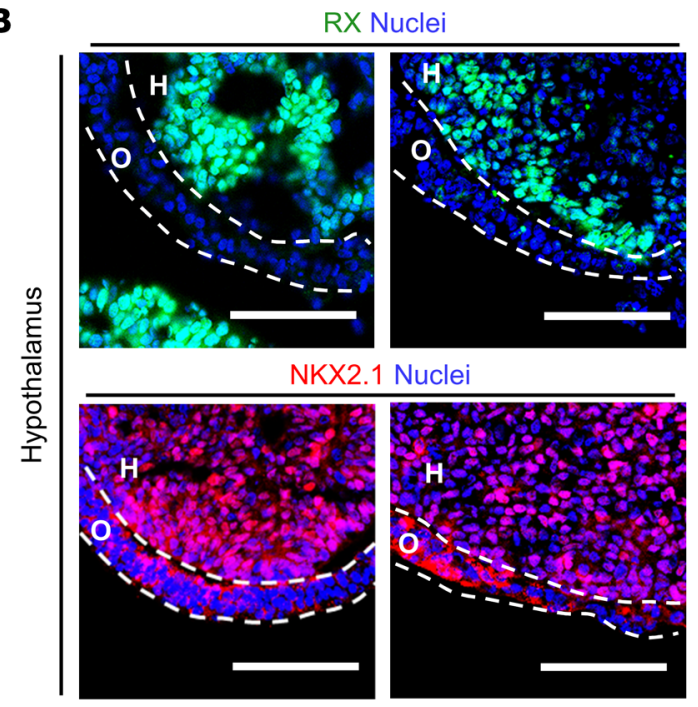

PITX1 EaCGalherin Nuclei

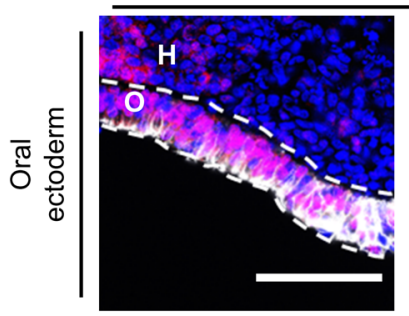

Control-iPSCs \#1

D

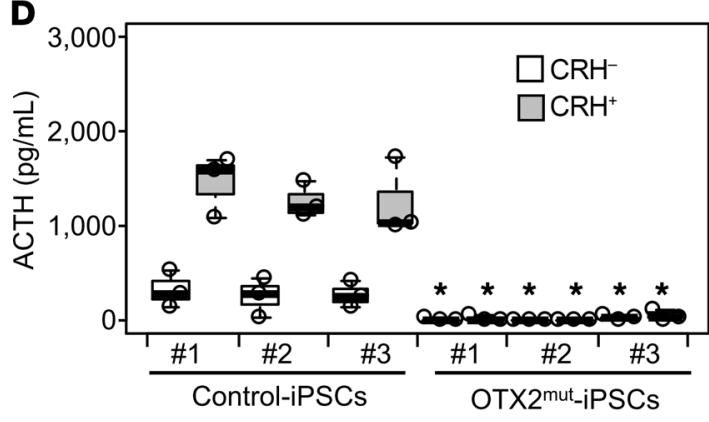

NANOG OCT3/4

E-cadherinNuclei
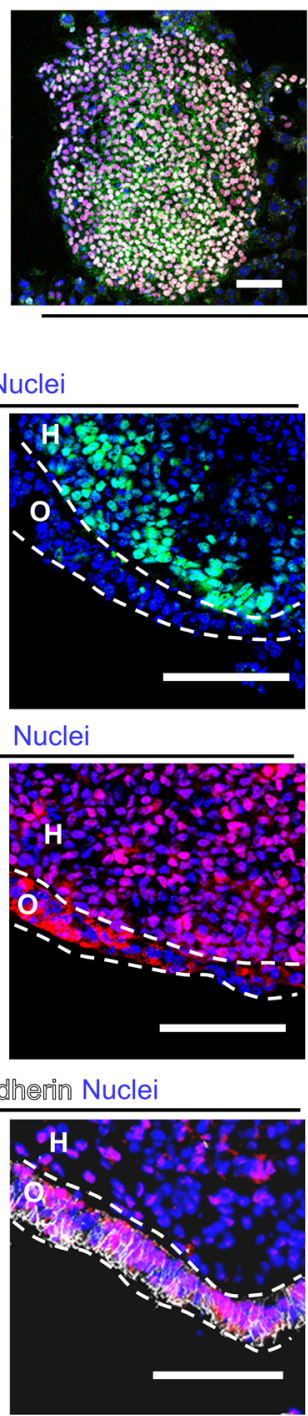

OTX2 ${ }^{\text {mut_iPSCs \#1 }}$
Undifferentiated markers
C

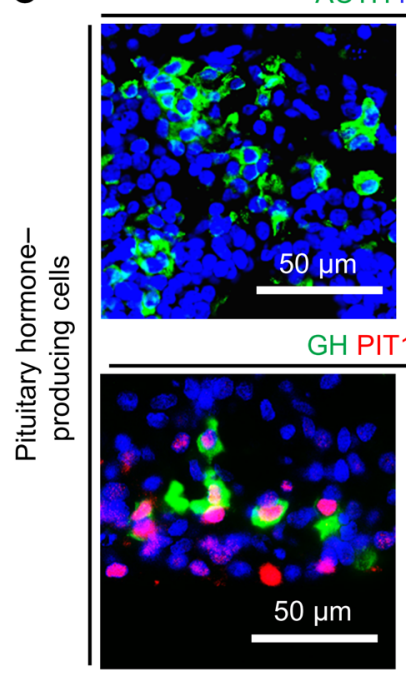

Control-iPSCs \#1
ALP staining

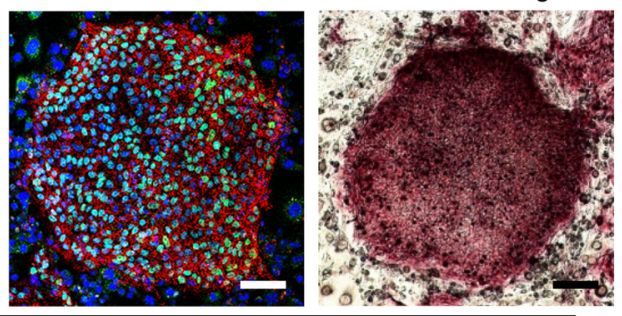

ACTH Nuclei
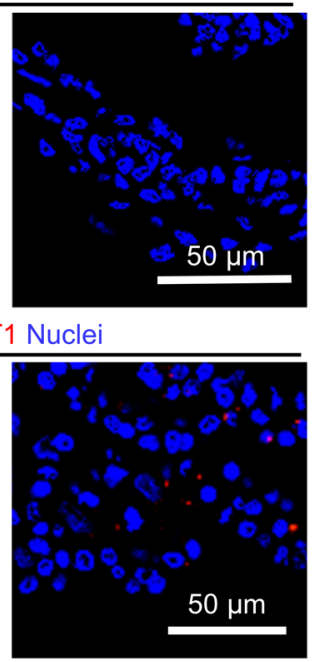

OTX2mut_iPSCs \#1

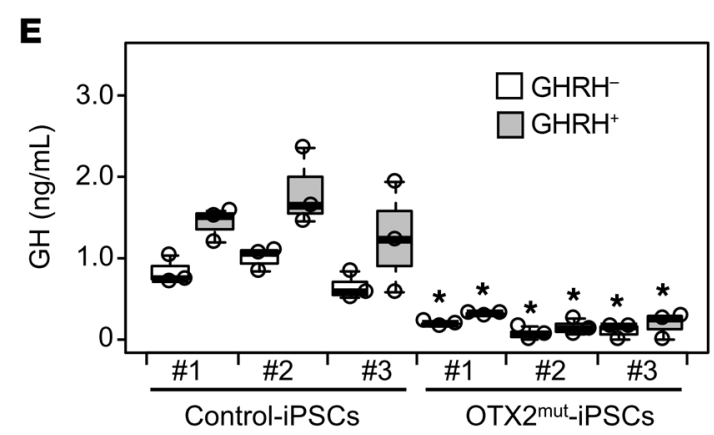

Figure 2. Establishment of patient-derived iPSCs and induction into the pituitary and hypothalamus in vitro. (A) Characterization of established patient-derived iPSCs. Phase-contrast image and immunostaining for the undifferentiated markers. ALP, alkaline phosphatase. (B) Immunostaining of the cell aggregates (day 40). The inside of the aggregates corresponded to the hypothalamus progenitor $\left(\mathrm{RX}^{+}\right.$and $\left.\mathrm{NKX} 21^{+}\right)$. The outside layer of the aggregates, indicated by dashed lines, corresponded to the oral ectoderm, which is the pituitary origin (PITX1+ and E-cadherin ${ }^{+}$). (C) Immunostaining of the cell aggregates at day 100 . Control-iPSCs differenti-

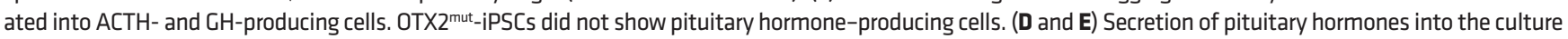
medium. Representative data from 3 independent experiments are shown. Control-iPSCs secreted a substantial amount of ACTH (D) or CH (E) and responded to CRH or GHRH stimulation, respectively. In contrast, OTX2 ${ }^{\text {mut }}$-iPSCs showed scant secretion of ACTH and $\mathrm{CH}$ with or without the stimulation. The line within the box indicates the median, the edge of the box represents the first and third quartiles, and the whiskers are the range of data; $n=3$ per group. ${ }^{*} P<0.05$, Kruskal-Wallis test $\left(\mathrm{ACTH}\right.$ without $\mathrm{CRH}, \chi^{2}=13.7$, degrees of freedom $[\mathrm{df}]=5, P=0.02$; $\mathrm{ACTH}$ with $\mathrm{CRH}, \chi^{2}=12.8, \mathrm{df}=5, P=0.03$; GH without $\mathrm{CHRH}, \chi^{2}=15.3, \mathrm{df}=5, P<0.01 ; \mathrm{CH}$ with $\mathrm{GHRH}, \chi^{2}=14.6, \mathrm{df}=5, P=0.01$ ) followed by post hoc Shirley-Williams test. Post hoc comparison with control-iPSCs no. 1 (far left) is presented. 0 , oral ectoderm; $\mathrm{H}$, hypothalamus progenitor. Scale bars without numbers: $100 \mu \mathrm{m}$. 
(Figure 1, D and E). Because it has been previously reported that mutations in OTX2 are associated with pituitary hypoplasia, these data strongly suggested that this variant was a disease-causing mutation. We then explored the detailed underlying mechanisms using patient-derived iPSCs.

Patient-derived iPSCs showed impaired differentiation into pituitary. To establish patient-derived iPSCs, peripheral leukocytes of the patient were reprogrammed using episomal vectors as previously described (25). We established 3 iPSC lines (OTX2 ${ }^{\text {mut }}$-iPSCs no. 1-3), and all of them showed embryonic stem cell-like morphology (Figure 2A) with normal karyotypes (Supplemental Figure $1 \mathrm{~A})$ and retained the mutation in OTX2 (Supplemental Figure 1B). These cells expressed undifferentiated markers (Figure $2 \mathrm{~A}$ and Supplemental Figure 1, C and D) and were able to differentiate into 3 germ layers in vitro (Supplemental Figure 1, E-G), indicating that these cells were pluripotent.

We then compared the ability of the 3 lines of OTX2 $2^{\text {mut }}$ iPSCs (no. 1-3) versus 3 lines of healthy control-derived iPSC lines (control-iPSCs no. 1-3) to differentiate into the pituitary using a 3D organoid culture method, in which the oral ectoderm and hypothalamus simultaneously developed in vitro as previously described (Supplemental Figure 2, A and B, and ref. 22). In this method, inside of the cell, aggregates differentiate into the hypothalamic progenitor, and the outer layer of the aggregates differentiate into the oral ectoderm that develops to anterior pituitary. At day 40, immunostaining showed that both control- and OTX2 ${ }^{\text {mut }}$-iPSCs expressed the hypothalamic markers $\mathrm{RX}$ and NKX2.1 and the oral ectoderm markers PITX1 and E-cadherin in a similar fashion (Figure 2B). The other 2 lines demonstrated similar results (Supplemental Figure 2C), indicating that the initial step of differentiation into pituitary was followed by both control-iPSCs and OTX2 $2^{\text {mut }}$-iPSCs in a similar fashion. After long-term culture for 100 days, control-iPSCs differentiated into adrenocorticotropic hormone-producing (ACTH-producing) and GH-producing cells (Figure 2C and Supplemental Figure 2D), consistent with a previous report (22); however, OTX2 ${ }^{\text {mut }}$-iPSCs did not give rise to these hormone-producing cells (Figure 2C and Supplemental Figure 2D). We further examined the ability to secrete hormones and the response to releasing of hormones (corticotropin-releasing hormone $[\mathrm{CRH}]$ and growth hormone-releasing hormone [GHRH]). Control-iPSC-derived cell aggregates secreted ACTH and $\mathrm{GH}$ in the basal status and responded to $\mathrm{CRH}$ and GHRH (Figure 2, D and E). However, in line with the results of immunostaining, ACTH and GH concentrations in the media were significantly lower in OTX2 ${ }^{\text {mut }}$-iPSC-derived than in control-iPSC-derived tissues, and responsiveness to the releasing hormones was also significantly impaired (Figure 2, D and E). These results clearly demonstrated that the ability of OTX $2^{\text {mut }}$-iPSCs to differentiate into the anterior pituitary was severely impaired.

To investigate the precise underlying mechanisms and which process was disrupted, we performed quantitative reverse transcriptase PCR (RT-PCR) for representative markers of the developmental process (Figure $3 \mathrm{~A}$ ) using mRNA extracted from the aggregates at days 0,20 , and 40 . Although there were no obvious differences in the expression of HESX1, SIX1, and PITX1, the expression of $L H X 3$ was significantly decreased in OTX2 ${ }^{\text {mut }}$-iPSCs (Figure $3 \mathrm{~B}$ ). In line with the results of mRNA analysis, immuno- staining clearly demonstrated a lack of LHX3 expression in the oral ectoderm layer derived from OTX2 $2^{\text {mut }}$-iPSCs (Figure $3 \mathrm{C}$ and Supplemental Figure 2C). In addition, the thickness of oral ectoderm was obviously decreased (Figure 3C and Supplemental Figure $2 \mathrm{C}$ ). These data indicated that the induction of pituitary progenitor cells $\left(\mathrm{LHX}^{+}\right)$from oral ectoderm $\left(\mathrm{PITX}^{+}, \mathrm{E}\right.$-cadherin $\left.{ }^{+}\right)$ was impaired in OTX2 ${ }^{\text {mut }}$-iPSCs.

It has been previously reported that mutations in $L H X 3$ cause pituitary hypoplasia with variable severity of pituitary dysfunction, from isolated GH deficiency to combined pituitary hormone deficiency (26). LHX3 plays an essential role in the cell expansion of pituitary progenitor cells, and $L h \times 3$-knockout mice exhibited enhanced apoptosis in the pituitary progenitor cells $(6,27,28)$. We then analyzed cell proliferation and apoptotic markers. Similar to the results from $L h \times 3$-knockout mice, although the number of Ki- $67^{+}$cells was not different (Figure 3, D and E), the number of apoptotic cells defined as cleaved caspase-3-positive cells was significantly increased in OTX2 ${ }^{\text {mut }}$-iPSCs compared with controliPSCs (Figure 3, F and G). Taken together, these data suggested that impaired expression of LHX3 caused enhanced apoptosis in the pituitary progenitor cells and resulted in impaired pituitary

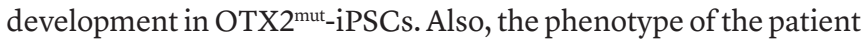
can be explained by the decreased expression in LHX3.

It has been reported that the promoter region of OTX2 possesses OTX2-binding consensus sites, suggesting the presence of auto-positive feedback $(29,30)$. To further understand the mechanisms, we examined OTX 2 mRNA expression level. Quantitative RT-PCR demonstrated that the OTX2 expression level decreased in OTX2 ${ }^{\text {mut }}$-iPSCs (Supplemental Figure 3A), suggesting auto-positive feedback of OTX2 and that the loss-of-function mutation also affected the OTX2 expression.

Because all 3 control-iPSC lines and 3 OTX2 ${ }^{\text {mut }}$-iPSC lines consistently demonstrated similar phenotypes (Supplemental Figure 2, C and D, and Supplemental Figure 3, B and C), we mainly used control-iPSC line no. 1 and OTX2 $2^{\text {mut }}$-iPSC line no. 1 for subsequent experiments.

Impaired function of hypothalamic OTX2 was responsible for LHX3 expression. It has been previously reported that OTX2 is expressed in both oral ectoderm and hypothalamus during mouse development (13). Indeed, OTX2 was expressed in both oral ectoderm (outer layer) and hypothalamus (inside the aggregates) in the iPSC-derived tissue (Supplemental Figure 4A). Interestingly, OTX2 localized almost exclusively in the nucleus in controliPSCs; however, it localized also in cytosol in OTX2 ${ }^{\text {mut-iPSCs }}$ (Supplemental Figure 4A). It has also been reported that OTX2 expression in hypothalamus is required for anterior pituitary development (15) and that some patients with OTX2 mutation demonstrated a deficiency in both anterior and posterior pituitary lobes (31). To evaluate OTX2 expression in oral ectoderm and hypothalamus in the organoid tissues, we separated the cells in oral ectoderm and hypothalamus by FACS using anti-E-cadherin (oral ectoderm marker) antibody (Figure 4A and Supplemental Figure 4, B and C) and performed quantitative RT-PCR for OTX2. The expression level of OTX2 was obviously predominant in hypothalamus compared with oral ectoderm (Figure 4B). These data suggested that hypothalamic OTX2 might play a pivotal role in anterior pituitary development. 
A

$$
\text { E7.5 }
$$

E9.5

E10.5

E11.5-13.5

E17.5
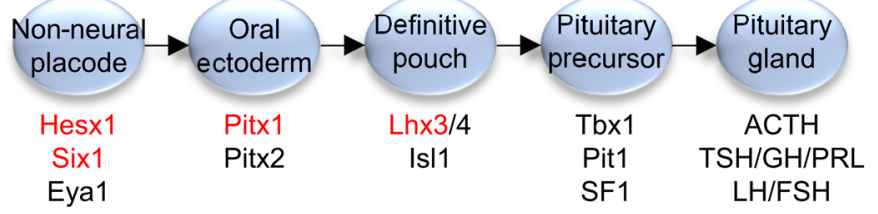

\section{B}
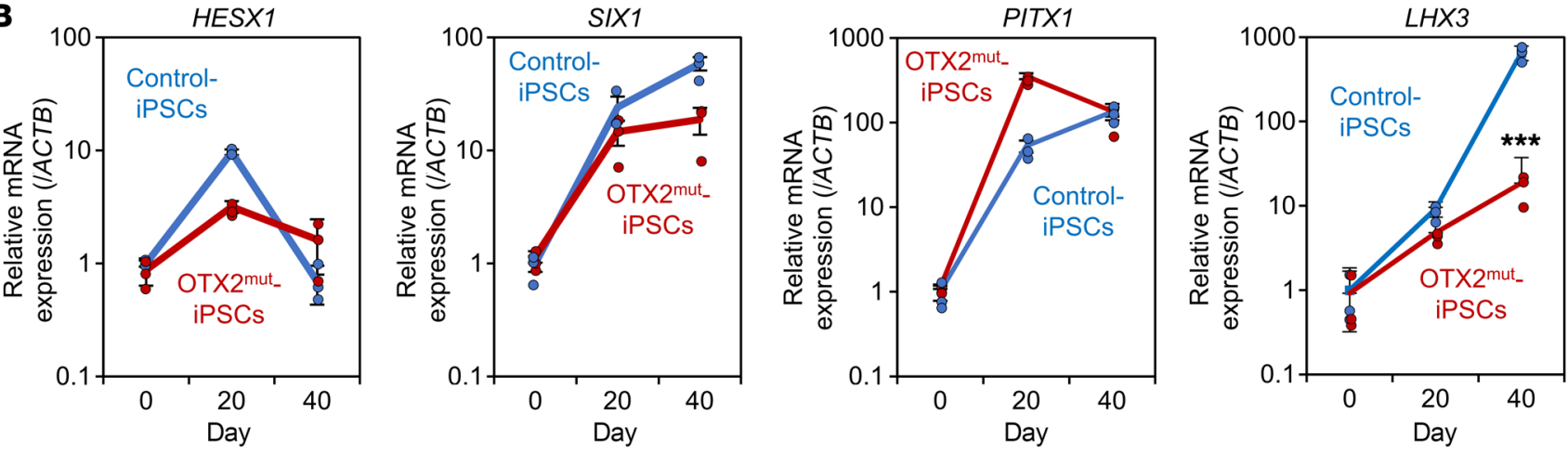

C

LHX3 RX Nuclei

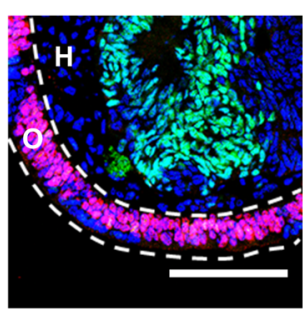

Control-iPSCs \#1

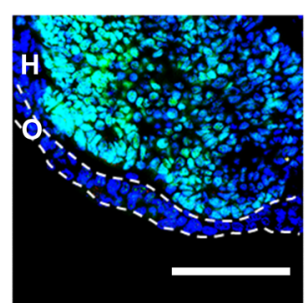

OTX2 ${ }^{\text {mut_iPSCs \#1 }}$

D

Ki-67 E-Gadherin Nuclei

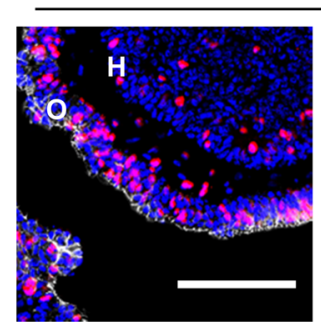

Control-iPSCs \#1

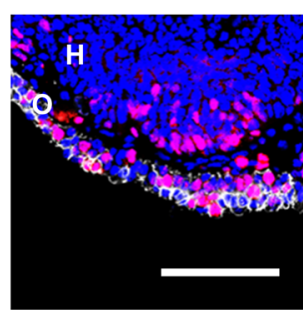

OTX2 $2^{\text {mut_iPSCs \#1 }}$

$\mathbf{F}$

C-Casp3 E-cadherin Nuclei

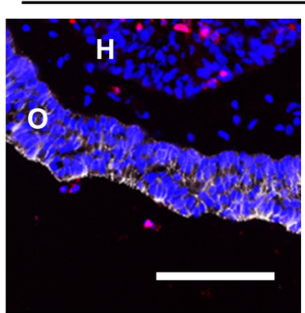

Control-iPSCs \#1

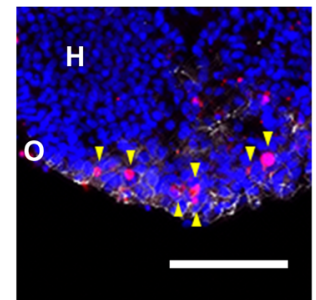

OTX2mut_iPSCs \#1

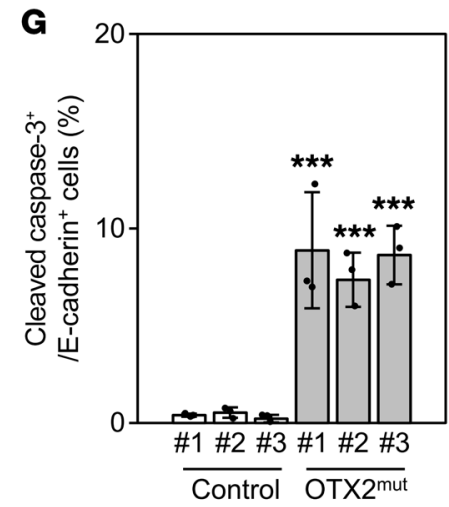

E

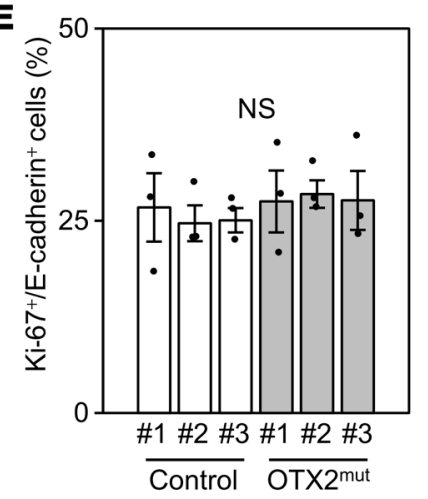

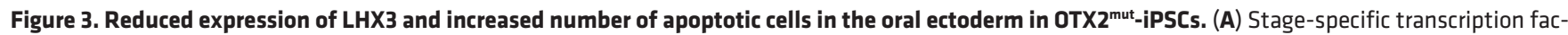
tors during pituitary differentiation in mouse development. (B) Quantitative RT-PCR analysis for representative markers of different developmental stages: non-neural placode, HESX1 and SIX1; oral ectoderm, PITX1; and pituitary progenitor, LHX3. Pituitary progenitor marker (LHX3) was significantly downregulated in OTX2 ${ }^{\text {mut }}$ iPSCs at day 40 . Representative data from 2 independent experiments are shown. Data show mean \pm SEM; $n=3$ per group. ${ }^{* * *} P<0.001$,

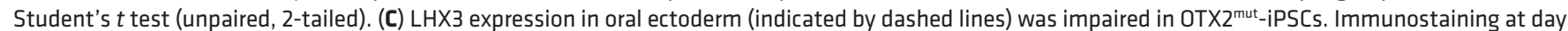
40. ( $\mathbf{D}$ and $\mathbf{E}$ ) Cell proliferation was evaluated by Ki-67 immunostaining. No significant difference in the number of Ki-67 and E-cadherin ${ }^{+}$cells was observed. Immunostaining at day 40. Representative data from 3 independent experiments are shown. Data show mean \pm SEM; $n=3$ per group; 1 -way ANOVA ( $F$ $=0.22, \mathrm{df}=5, P=0.95)$. ( $\mathbf{F}$ and $\mathbf{G})$ Abundance of apoptotic cells was evaluated by cleaved caspase-3 immunostaining. The number of apoptotic cells was significantly increased in oral ectoderm (E-cadherin ${ }^{+}$) derived from OTX2 ${ }^{\text {mut }}$-iPSCs. Immunostaining at day 40 . Representative data from 3 independent experiments are shown. Data show mean $\pm \mathrm{SEM} ; n=3$ per group. ${ }^{* *} P<0.001,1$-way ANOVA $(F=25.9, \mathrm{df}=5, P<0.001)$ followed by post hoc Dunnett's test. Post hoc comparison with control-iPSCs no. 1 (far left) is presented. O, oral ectoderm; H, hypothalamus progenitor. Scale bars: $100 \mu \mathrm{m}$. 
A

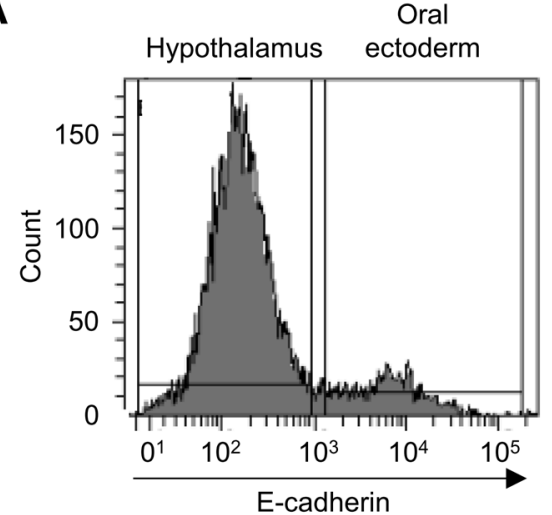

B

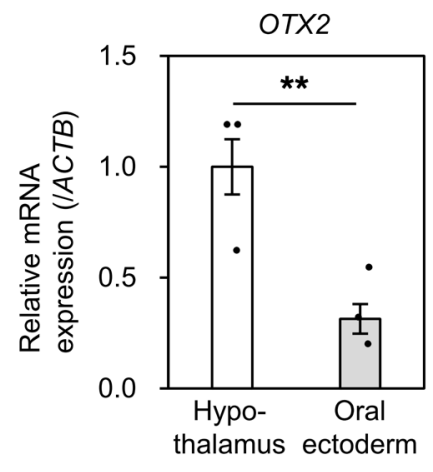

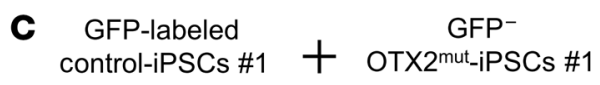

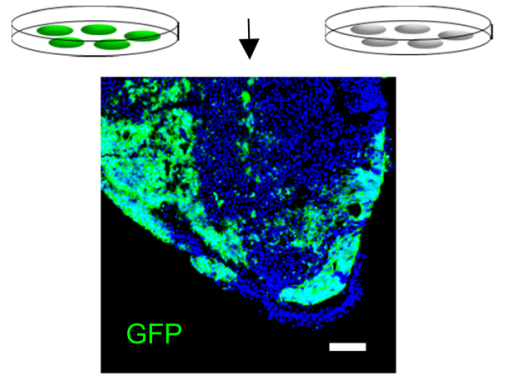

Mosaic aggregate

D

LHX3 GFP E-Cad|herinn Nuclei
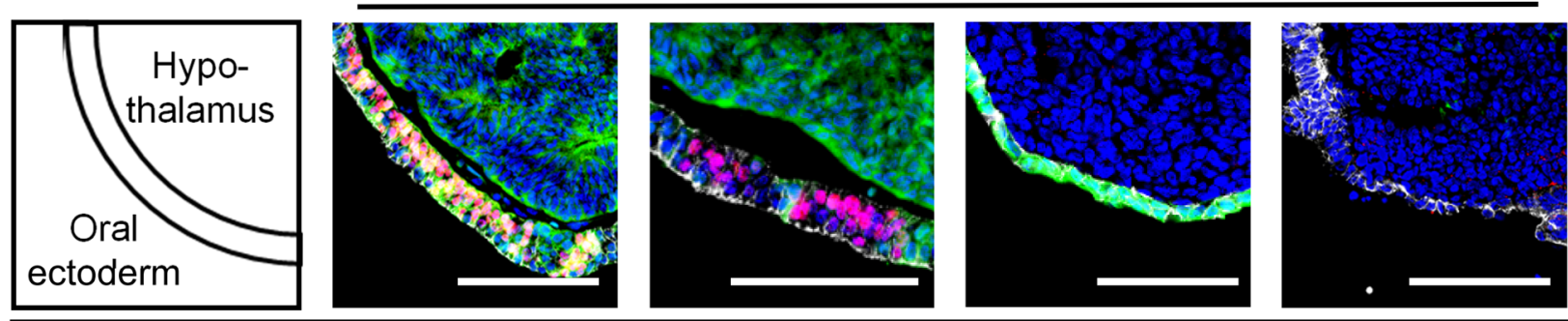

\begin{tabular}{|c|c|c|c|c|}
\hline Hypothalamus & Control & Control & OTX2mut & OTX2mu \\
\hline Oral ectoderm & Control & OTX2mut & Control & OTX2 \\
\hline LHX3 expression & + & + & - & - \\
\hline
\end{tabular}

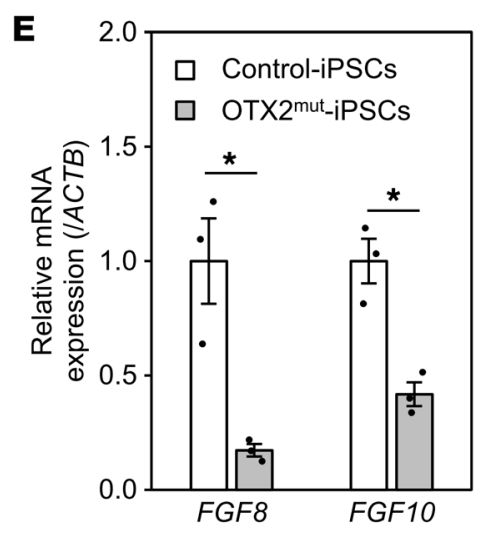

$\mathbf{F}$

FGF8 Nuclei

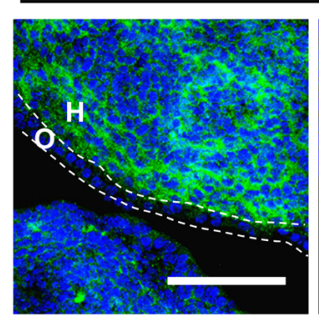

Control-iPSCs \#1

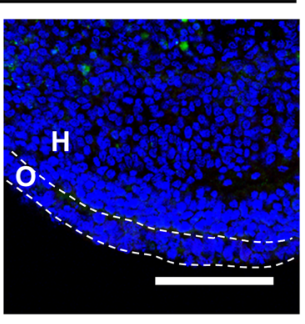

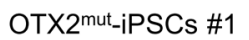

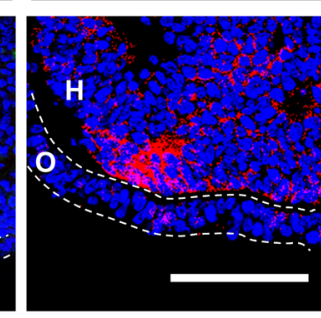

Control-iPSCs \#1
FGF10 Nuclei

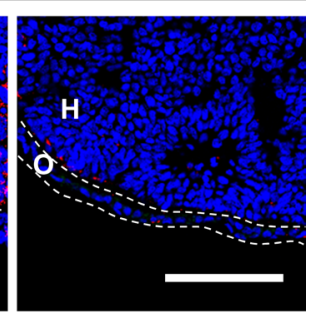

OTX2mut_iPSCs \#1
G

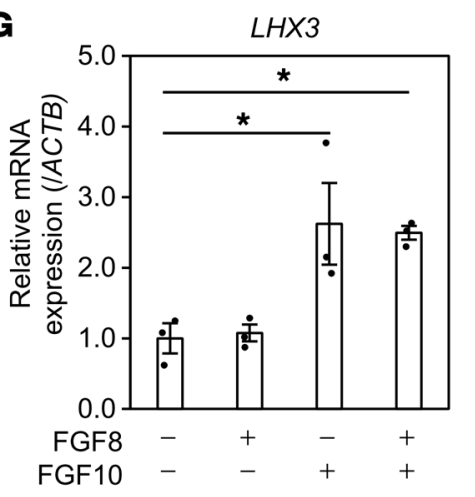

H

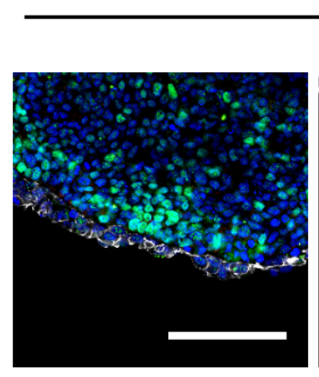

Vehicle

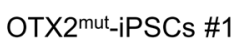

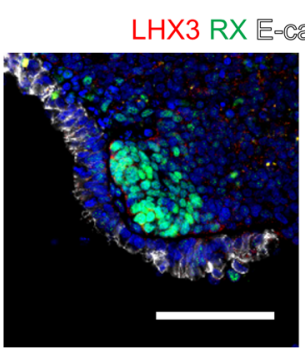

FGF8

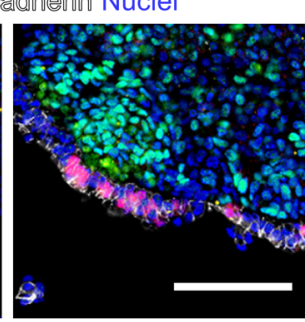

FGF10

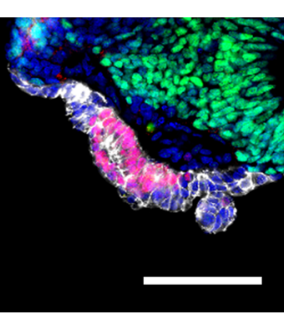

FGF8/FGF10 
Figure 4. Decreased expression of hypothalamic FGF10 was responsible

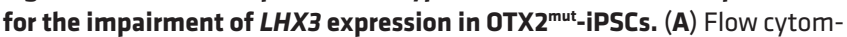
etry analysis for E-cadherin in control-iPSC-derived aggregates (day 16). FACS-sorted E-cadherin ${ }^{-}$and E-cadherin ${ }^{+}$cells were defined as hypothalamus and oral ectoderm cells, respectively. (B) Quantitative RT-PCR of OTX2 in FACS-sorted hypothalamus and oral ectoderm (day 16-30). OTX2 expression was higher in hypothalamus than in oral ectoderm. Data show mean \pm SEM; $n=3$ per group. ${ }^{* *} P<0.01$, Student's $t$ test (unpaired, 2 -tailed). (C) Coculture of GFP-labeled control-iPSCs and OTX2 ${ }^{\text {mut }}$ iPSCs produced chimeric aggregates. (D) The chimeric aggregates were induced for pituitary differentiation. We observed 4 combinations of hypothalamus and oral ectoderm according to the origin. The LHX3 expression in oral ectoderm was observed only in the presence of control-iPSC-derived hypothalamus. ( $E$ and $\mathbf{F}$ ) Quantitative RT-PCR and immunostaining of FGF8 and FGF10 in iPSC-derived tissues. The expression of FGF8 and FGF10 was decreased at both the mRNA and the protein level in the hypothalamus of OTX2 ${ }^{\text {mut }}$-iPSCs (day 40). Representative data from 2 independent experiments are shown. Data show mean \pm SEM; $n=3$ per group. ${ }^{*} P<0.05$, Student's $t$ test (unpaired, 2-tailed). (G and $\mathbf{H}$ ) Quantitative RT-PCR and immunostaining of LHX3 in IPSC-derived tissue treated with or without recombinant FGF8 and FGF10. FGF8 $(100 \mathrm{ng} / \mathrm{mL})$ and/or FGF10 $(50 \mathrm{ng} / \mathrm{mL})$ were added in culture medium from day 9 to day 40 . Not FGF8 but FGF10 treatment restored LHX3 expression in OTX2 ${ }^{\text {mut }}$-iPSC-derived oral ectoderm at both the mRNA and the protein level (day 40). Representative data from 2 independent experiments are shown. Data show mean $\pm \mathrm{SEM} ; n=3$ per group. ${ }^{*} P<0.05$, 1 -way ANOVA ( $F=7.67, \mathrm{df}=3, P<0.01)$ followed by post hoc Dunnett's test. Post hoc comparison with control-iPSCs no. 1 (far left) is presented. 0 , oral ectoderm; $\mathrm{H}$, hypothalamus progenitor. Scale bars: $100 \mu \mathrm{m}$.

To clarify which OTX2 expressed in the oral ectoderm or the hypothalamus plays an essential role in the development of pituitary, we performed a chimera formation experiment using controland OTX2 ${ }^{\text {mut }}$-iPSCs. We cocultured GFP-labeled control-iPSCs (Figure $4 \mathrm{C}$ and Supplemental Figure $5, \mathrm{~A}^{-} \mathrm{C}$ ) and OTX2 ${ }^{\text {mut }}$-iPSCs, and induced differentiation (Figure $4 \mathrm{C}$ ). These culture conditions successfully developed the pituitary and hypothalamus with a mosaic formation (Figure 4C and Supplemental Figure 5D). Inter-

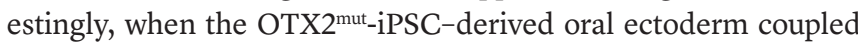
with control-iPSC-derived hypothalamus, LHX3 expression was restored in OTX2 ${ }^{\text {mut }}$-iPSCs (Figure $4 \mathrm{D}$ and Supplemental Figure $5 \mathrm{E}$ ). In contrast, when coupled with OTX $2^{\text {mut }}$-iPSC-derived hypothalamus, even control-iPSC-derived oral ectoderm showed diminished LHX3 expression, clearly indicating that hypothalamic OTX2 is essential for the LHX3 expression in the oral ectoderm (Figure 4D). These data indicated that control-iPSC-derived hypothalamus rescued LHX3 expression in OTX $2^{\text {mut }}$-iPSC-derived oral ectoderm, suggesting that some factors present in hypothalamus required for the differentiation of pituitary progenitor cells might be absent in OTX2 ${ }^{\text {mut }}$-iPSC-derived tissue.

Roles of FGFs from hypothalamus in pituitary development. During pituitary development, several growth/differentiation factors, including BMP4, FGF8, FGF10, SHH, and WNT5a, from surrounding tissues including hypothalamus play an essential role (32). Among them, close interaction between OTX2 and FGF has been reported previously. Otx2 regulates Fgf expression and plays an important role in early brain regionalization $(33,34)$. On the other hand, inhibition of FGF signaling upregulates OTX2 expression in human embryonic stem (ES) cells (35). In addition, hypothalamic Shh and Fgf signaling is required for the cell specification of $\operatorname{Lhx} 3^{+}$ pituitary progenitor cells $(36,37)$. Therefore, we explored whether

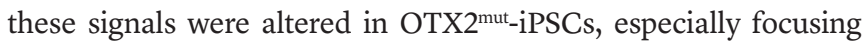
on FGF and SHH signals. We evaluated these signals by immunostaining for FGF8, FGF10, SHH, and GLI1 and quantitative RT-PCR for ID1 (a readout of BMP signal), FGF8, FGF10, SHH, GLI1, GLI2 (readouts of SHH signal), and AXIN2 (a readout of WNT signal)

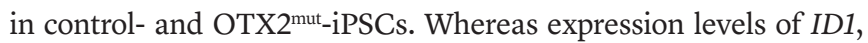
SHH, GLI1, GLI2, and AXIN2 were not significantly different between control- and OTX2 ${ }^{\text {mut }}$-iPSCs (Supplemental Figure 6, A and B), expression of both $F G F 8$ and FGF1O significantly decreased in OTX2 ${ }^{\text {mut }}$-iPSCs (Figure 4, E and F, and Supplemental Figure 3D). In addition, FGF8 and FGF1O were expressed predominantly in hypothalamus compared with oral ectoderm (Supplemental Figure 7A), suggesting the importance of the expression in hypothalamus.

To investigate the role of FGFs in this pituitary developmental model, we treated control-iPSCs with FGF receptor inhibitor (PD173074). Inhibition of FGF signaling using PD-173074 significantly decreased $L H X 3$ expression in the oral ectoderm derived from control-iPSCs at the mRNA (Supplemental Figure 7B) and protein levels (Supplemental Figure 7C). In addition, induction into pituitary hormone-producing cells was disrupted by PD-173074 treatment (Supplemental Figure 7D). To clarify which FGF plays an essential role in this process, we performed a rescue experiment. Addition of recombinant FGF8 into the culture media did not rescue the LHX3 expression (Figure 4G), although FGF8 increased the thickness of oral ectoderm (Figure $4 \mathrm{H}$ and Supplemental Figure 7E). However, in contrast to FGF8, addition of recombinant FGF10 clearly restored the LHX3 expression in the oral ectoderm

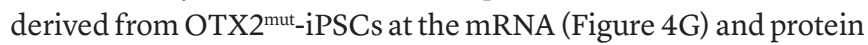
levels (Figure 4H). Compatible with these results, FGF10 immunostaining of chimeric aggregates demonstrated that FGF10 was mainly expressed in the control-iPSC-derived hypothalamus region $\left(\mathrm{GFP}^{+}\right)$(Supplemental Figure $7 \mathrm{~F}$ ), supporting the hypothesis that FGF10 expressed in $\mathrm{GFP}^{+}$control-iPSC-derived hypothalamus rescued the LHX3 expression in OTX2 ${ }^{\text {mut }}$-iPSCs. Taken together, these results indicated that OTX2-dependent expression of FGF10 in hypothalamus plays an essential role in LHX3 expression in the pituitary progenitor cells.

CRISPR/Cas9-based gene correction of the mutation in OTX2 and deletion of OTX2. To verify that the mutation in OTX2 was responsible for the impaired phenotype of iPSCs and for the disease pathogenesis, we repaired the mutation in OTX2 using CRISPR/Cas9 genome editing in OTX2 ${ }^{\text {mut }}$-iPSCs (Supplemental Figure 8A). Two established cell lines (no. 1 and no. 2) retained ES celllike morphology (Supplemental Figure 8B). We then characterized the ability of these cells to differentiate into pituitary. Compared with the parental OTX $2^{\text {mut }}$-iPSCs, OTX $2^{\text {mut }}$-repaired-iPSCs demonstrated a restoration of LHX3 expression during the pituitary differentiation (Figure 5, A and B). The expression of FGF8, FGF10, and OTX2 (Figure 5C and Supplemental Figure 8, C, E, and F) was increased. The number of apoptotic cells in oral ectoderm were significantly decreased by the correction (Supplemental Figure 8D). Furthermore, production of ACTH and GH was also restored (Figure 5D), and OTX2 ${ }^{\text {mut }}$-repaired-iPSCs showed a response to the releasing hormones (CRH and GHRH) (Figure 5, E and F), demonstrating an ability to differentiate into the pituitary similar to that of control-iPSCs. These results clearly indicated that the mutation in OTX2 was responsible for the impaired pituitary development. 
A

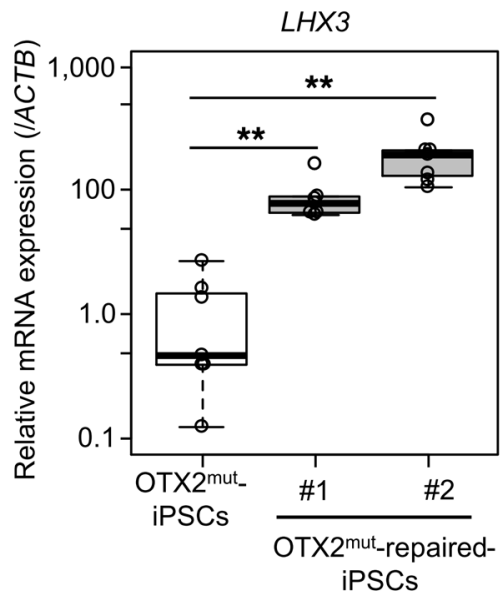

B

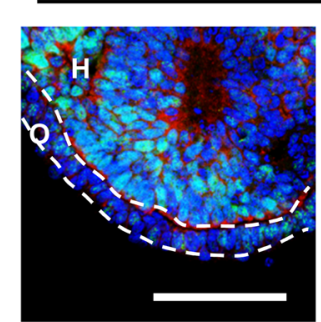

OTX2 $2^{\text {mut_iPSCs }}$

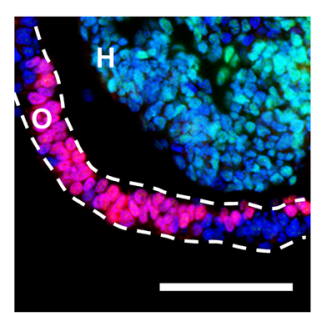

\#1

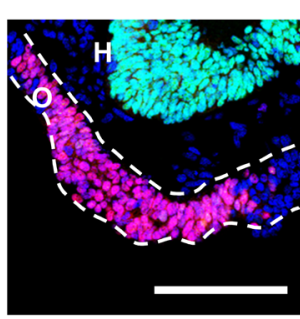

\#2

OTX2 $2^{\text {mut_repaired-iPSCs }}$

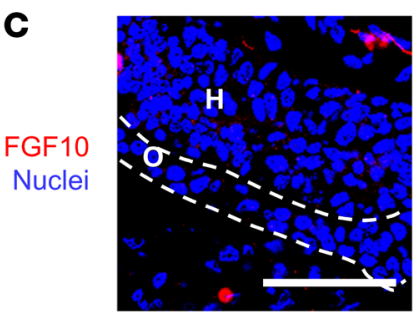

OTX2mut_iPSCs
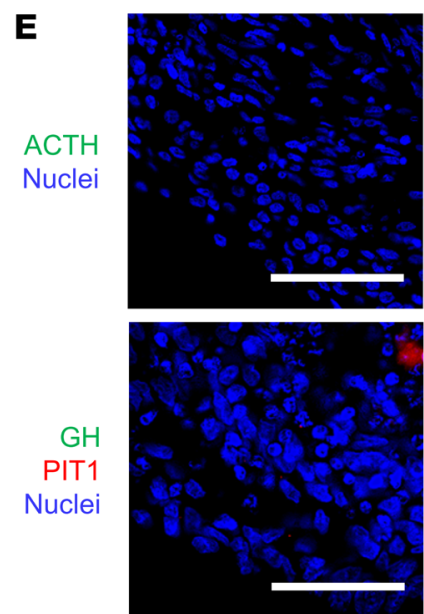

OTX2mut_iPSCs

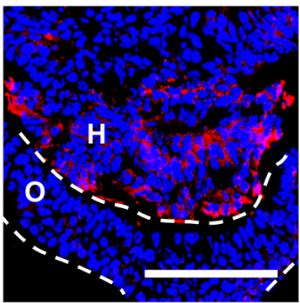

\#1

OTX2 $2^{\text {mut_repaired-iPSCs }}$
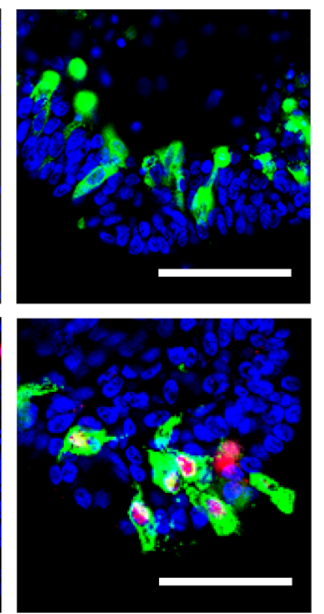

\#1
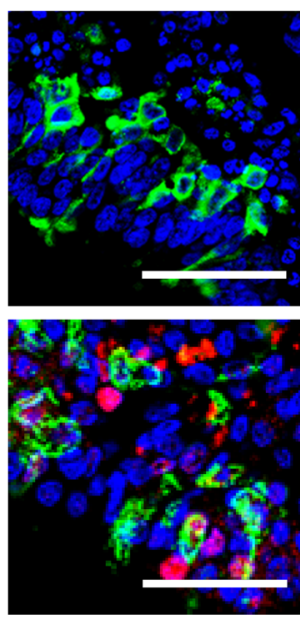

\#2
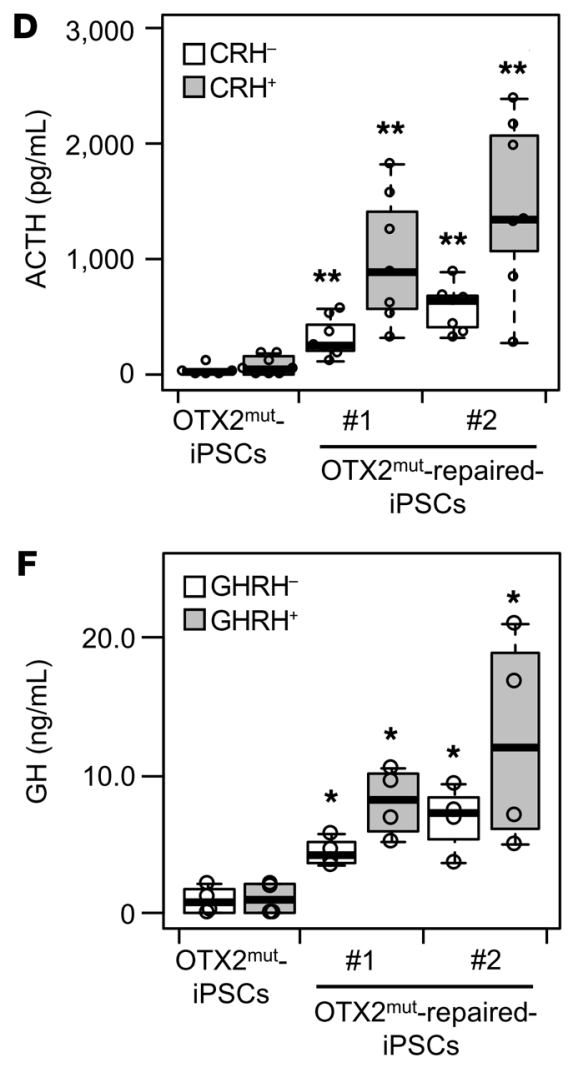

OTX2mut_repaired-iPSCs

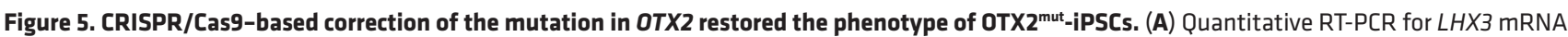
expression in OTX2 ${ }^{\text {mut }}$-iPSCs and OTX $2^{\text {mut }}$-repaired-iPSCs at day 40 . Representative data from 4 independent experiments are shown. The line within the box indicates the median, the edge of the box represents the first and third quartiles, and the whiskers are the range of data excluding outliers; $n=7$ per group. ${ }^{*} P<0.01$, Kruskal-Wallis test $\left(\chi^{2}=16.8, \mathrm{df}=2, P<0.001\right.$ ) followed by post hoc Steel's test. Post hoc comparison with OTX2mut_iPSCs (far left) is presented. (B) Immunostaining of OTX2 ${ }^{\text {mut }}$ - and OTX2 ${ }^{\text {mut }}$-repaired-iPSCs. OTX2 ${ }^{\text {mut }}$-repaired-iPSCs restored LHX3 expression in oral ectoderm (indicated with dashed lines) (at day 40). (C) FGF10 expression in the hypothalamus was restored in OTX2 ${ }^{\text {mut }}$-repaired-iPSCs (at day 40). (D) The induction of ACTH- and $\mathrm{GH}$-producing cells was restored in OTX2 ${ }^{\text {mut }}$-repaired-iPSCs (at day 100). (E and F) The ability to secrete hormones and the response to releasing hormones were restored in OTX $2^{\text {mut }}$-repaired-iPSCs at day 100 . Representative data from 4 independent experiments are shown. The line within the box indicates the median, the edge of the box represents the first and third quartiles, and the whiskers are the range of data excluding outliers; $n=7$ per group for $\mathrm{ACTH}, n=$ 4 per group for $\mathrm{GH}$; . ${ }^{*} P<0.05$, ${ }^{*} P<0.01$, Kruskal-Wallis test $\left(\mathrm{ACTH}\right.$ without $\mathrm{CRH}, \chi^{2}=15.0, \mathrm{df}=2, P<0.001 ; \mathrm{ACTH}$ with $\mathrm{CRH}, \chi^{2}=14.0, \mathrm{df}=2, P<0.001 ; \mathrm{GH}$ without GHRH, $\chi^{2}=8.34, \mathrm{df}=2, P=0.015$; GH with GHRH, $\chi^{2}=7.54$, df $=2, P=0.023$ ) followed by post hoc Steel's test. Post hoc comparison with OTX2mut iPSCs (far left) is presented. 0 , oral ectoderm; $\mathrm{H}$, hypothalamus progenitor. Scale bars: $100 \mu \mathrm{m}$. 
Lastly, to investigate whether haploinsufficiency of OTX2 actually causes pituitary hypoplasia, we performed heterozygous and homozygous deletion of OTX2 in control-iPSCs no. 1 using CRISPR/Cas9 genome editing (Supplemental Figure 9A). We named established heterozygous and homozygous OTX2-knockout cells OTX2 $2^{\text {WT/insFs }}$-iPSCs and OTX2 $2^{\text {delFS/insFs }}-i P$ SCs, respectively (Figure 6A). These lines retained normal morphology of iPSCs (Supplemental Figure 9B) and comparable expression of the undifferentiated markers OCT4 and NANOG with the parental iPSCs (Supplemental Figure 9C). Immunoblotting analysis demonstrated a gene dosage-dependent OTX2 protein expression in the differentiated OTX2 ${ }^{\mathrm{WT} / \mathrm{insF}}$ - and OTX2 $2^{\text {delFs/insFs }}$-iPSCs (Supplemental Figure 9D). We then characterized the ability of OTX2 $2^{\mathrm{WT} / \text { insFs }}$ - and OTX2 $2^{\mathrm{delFS} / \mathrm{insF}}-\mathrm{iPSCs}$ to differentiate into the pituitary. Both OTX2 ${ }^{\mathrm{WT} / \mathrm{insF}}$ - and OTX$2^{\text {delFs/insFS }}-i P S C s$ exhibited impaired ability to differentiate, and expression of $L H X 3, O T X 2, R X, F G F 8$, and FGF1O was significantly decreased (Figure 6B and Supplemental Figure 10, A-C). Immunostaining also demonstrated reduced expression of LHX3 (Figure 6C) and increased abundance of apoptotic cells in the oral ectoderm (Figure 6D and Supplemental Figure 10D), and decreased expression of FGF10 in the hypothalamus (Figure 6E). Furthermore, these lines exhibited impaired differentiation into pituitary hormone-producing cells in long-term culture (Supplemental Figure 10, E and F). These results demonstrated that both heterozygous and homozygous OTX2 defects could be responsible for the impaired pituitary development and supported that the heterozygous OTX2 mutation (R127W) resulted in the patient's phenotype through haploinsufficiency.

The phenotypes of OTX $2^{\text {WT/insFs }}$ - and OTX $2^{\text {delFs/insFs }}-i P S C s$ were similar to that of OTX2 ${ }^{\text {mut }}$-iPSCs; however, there were several differences. Hypothalamic RX protein expression was scarcely observed in OTX2 $2^{\text {WT/insFs }}$ - and OTX2 $2^{\text {delFs/insFs }}-i P S C s$ (Figure 6C and Supplemental Figure 10B), whereas OTX2 ${ }^{\text {mut }}$-iPSCs retained RX protein expression (Figure 2B and Supplemental Figure 2C), indicating that OTX2 ${ }^{\mathrm{WT} / \mathrm{insF}}$ - and OTX2 $2^{\text {delFs/insFs }}-i P S C s$ had a more severe phenotype compared with OTX2 $2^{\text {mut }}$-iPSCs. This could be explained by the observation that the function of mutant OTX2 was partially impaired (Figure 1, D and E). In addition, these results also imply that the threshold level of OTX2 function required for development may be different between hypothalamus and pituitary; pituitary is more sensitive to the loss of function in OTX2.

\section{Discussion}

The pathogenesis of $\mathrm{CPH}$ remains largely unknown. Even using exome sequencing analysis, gene abnormalities have been identified in fewer than $20 \%$ of patients (4). Furthermore, the underlying mechanisms have not been fully understood because of a lack of a human model. In this study, we established an in vitro disease model of $\mathrm{CPH}$ using patient-derived iPSCs. iPSCs derived from the patient harboring mutation in OTX2 retained the potential to differentiate into oral ectoderm (PITX1 ${ }^{+}$and E-cadherin ${ }^{+}$) but exhibited a severely impaired pituitary differentiation. The patient-derived iPSCs exhibited a reduced expression of LHX3 and enhanced apoptosis compared with control-iPSCs during differentiation. In addition, we demonstrated that hypothalamic OTX2 was essential for FGF10 expression and FGF10 played an important role in the expression of LHX3 in pituitary progenitor cells (Figure 6F). Furthermore, we showed that mutation in OTX2 was responsible for the occurrence of $\mathrm{CPH}$ using mutation-repaired patient-derived iPSCs. In some patients with OTX2 mutation, eye and craniofacial defects are observed with pituitary defect (4). These data suggest that the pituitary defect may be attributable not to intrinsic defect but to structural defect including hypothalamus. However, our results suggested that the OTX2/ FGF10/LHX3 pathway also plays an important role in pituitary development, although the pituitary hypoplasia in the patient might be attributable in part to a secondary effect of structural defect.

In this study, we showed that the model for $\mathrm{CPH}$ based on patient-derived iPSCs is useful for analyzing the underlying mechanisms of the disease. This method enabled in vitro pituitary and hypothalamus self-organization along with essential interaction of these tissues, which recapitulates pituitary developmental process. Although many knockout mouse models have been previously used for understanding pathogenesis (5), there have been substantial species differences in terms of mode of inheritance and phenotypes (6), and sometimes it is difficult to elucidate the detailed mechanisms. During genetic analyses in humans, most of the patients do not reveal any plausible mutations (4), and even if the candidate gene variant is identified, it would be difficult to prove that the mutation was responsible for the disease in many cases (38). In addition, many $\mathrm{CPH}$ patients demonstrate sporadic onset, low penetration, and variable phenotypes with the same mutation. Although polygenic inheritance may explain its pathogenesis $(39,40)$, as has been previously shown in Kallman syndrome (41), it would be difficult to prove the precise pathogenesis. Our model using patient-derived iPSCs has several advantages. First, patient-derived iPSCs maintain all the gene variants, and theoretically, it is possible to prove which variant is responsible. Second, it is easy to identify which step in the pituitary development process is impaired and to intervene during the differentiation process to clarify the underlying mechanisms. Third, the hypothalamic-pituitary interaction during the development process that is essential for pituitary organogenesis can be recapitulated using this method. Finally, there are no species differences when analyzing a human disease. In this regard, an in vitro pituitary differentiation model based on patient-derived iPSCs is a promising tool in this field.

Interaction between oral ectoderm and hypothalamus progenitor is essential for embryonic pituitary development (32). In particular, factors from hypothalamus and surrounding tissues including BMP4, SHH, FGF8, FGF10, and WNT5a play a pivotal role (36, 37, 42). For example, it has been reported that SHH, FGF8, and FGF10 from hypothalamus synergistically upregulate LHX3 expression in the pituitary progenitor (37). Previously, it has been reported that neural ectoderm-specific deletion of Otx2 delays anterior pituitary development because of impaired cell proliferation, and it was speculated that decreased expression of Fgf10 might play an important role (15); however, the precise underlying mechanisms have not been clarified. In this study, we demonstrated that hypothalamic OTX2 mediates oral ectodermal LHX3 expression, in part by regulating hypothalamic FGF10, which is required for pituitary progenitor cell maintenance. These data are compatible with the reports that FgflO-deficient mice showed pituitary hypoplasia (43), that mice deficient in Fgfreceptor $2 \mathrm{IIIb}$ (receptor of Fgf10) exhibited a severe defect of the pituitary gland (44), and that $L h x 3$-deficient 
A

OTX2 ${ }^{\text {WT/insFS-iPSCs }}$
Initiation codon
DSB
PAM

AACCTTAGCATGATGTCTTATCTTAAGCAACCGCCTTACGCAGTCAATGGGCTGAGTCTGACCACTTCGGGT: WT AACCTTAGCATGATGTCTTATCTTAAGCAACCGCCTTACGCAGTCAATGGGCTGAGTCTGACCACTTCGGGT: Out of frame OTX2delFS/insFs_iPSCs

Initiation codon
AACCTTAGCATGATG -14 deletion

B

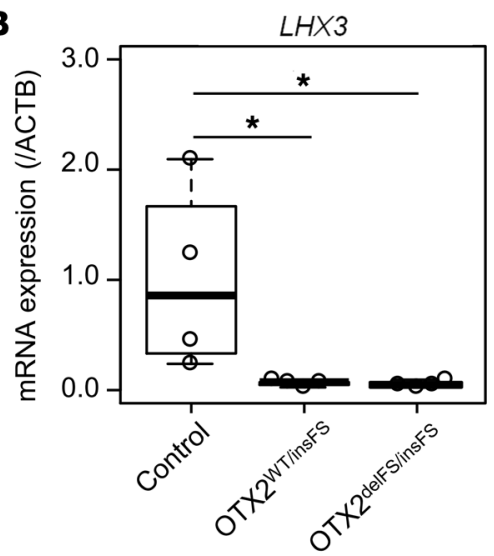

C

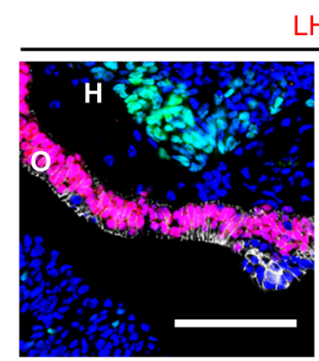

Control-iPSCs \#1

LHX3 RX E-Gadherion Nuclei

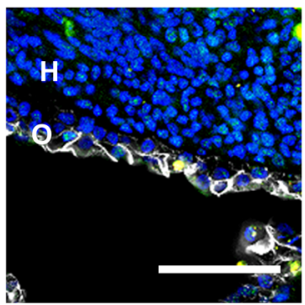

OTX2WT/insFS

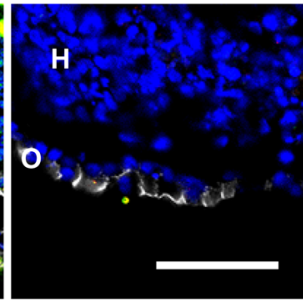

OTX2 ${ }^{\text {delFS/insFS }}$

D

C-Casp3 Eேc.adherin Nuclei

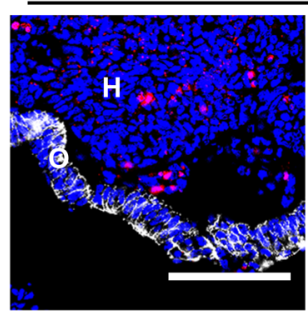

Control-iPSCs \#1

E
Control-iPSCs \#1
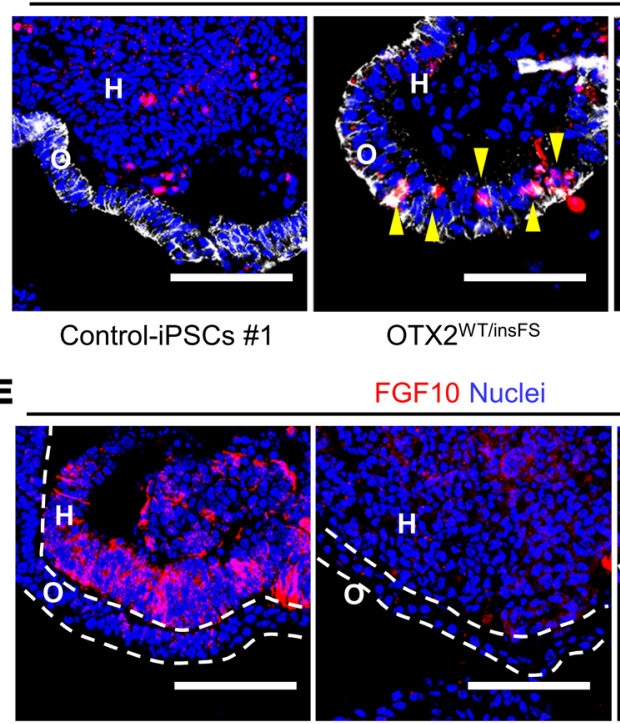

OTX2 ${ }^{\text {WT } / \text { insFS }}$

FGF10 Nuclei

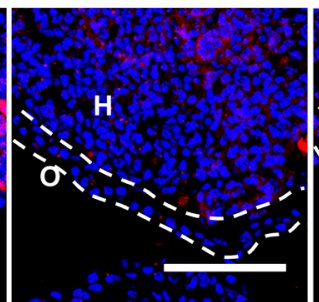

OTX2WT/insFs

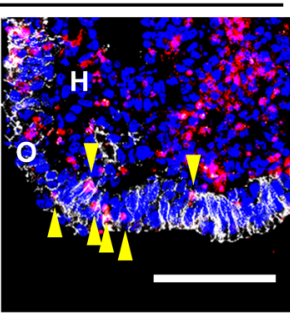

OTX2 $2^{\text {delFS/insFs }}$
$\mathbf{F}$

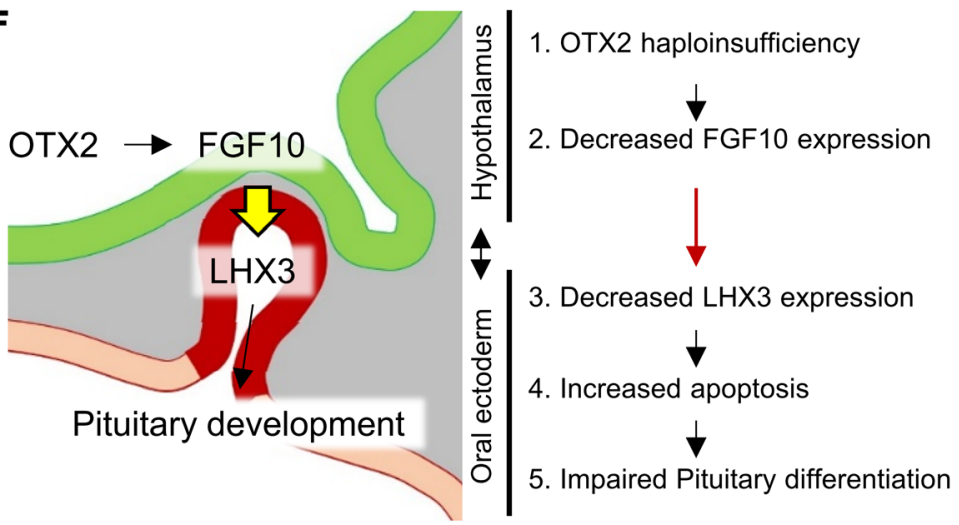

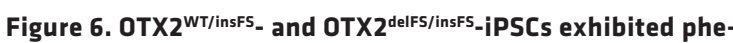
notype similar to that of patient-derived iPSCs. (A) Sequence analysis of OTX2 in OTX2 $2^{\text {WT/insFs }}$ - and OTX $2^{\text {delF5/insFs }-i P S C s . ~ D S B, ~}$ double-strand break; PAM, protospacer adjacent motif. (B) Quantitative RT-PCR for $L H X 3$ mRNA expression in control-,

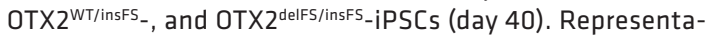
tive data from 4 independent experiments are shown. The line within the box indicates the median, the edge of the box represents the first and third quartiles, and the whiskers are the range of data; $n=4$ per group. ${ }^{*} P<0.05$, Kruskal-Wallis test $\left(\chi^{2}=7.54, \mathrm{df}=2, P=0.02\right.$ ) followed by post hoc Steel's test. (C) Immunostaining of control-, OTX2 ${ }^{\text {WT/insFs }}$,

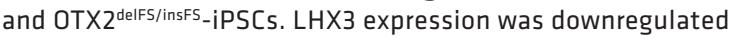
in OTX2 $2^{\mathrm{WT} / \text { insFs }}$ - and OTX2 $2^{\text {delFs/insFs }}$-iPSCs (at day 40). (D) The number of apoptotic cells, stained with cleaved caspase-3 cells (indicated by arrowheads), was increased in OTX2 $2^{\text {WT/insFs }}$

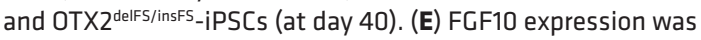

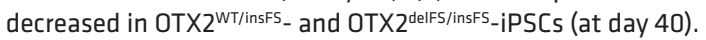
These phenotypes were similar to that of OTX2 $2^{\text {mut }}$-iPSCs. 0 , oral ectoderm; $\mathrm{H}$, hypothalamus progenitor. Scale bars: $100 \mu \mathrm{m}$. (F) Schematic image of the role of OTX2 in pituitary development and the underlying mechanisms of the mutation in OTX2. OTX2 is necessary for the expression of FGF10 in the hypothalamus. FGF10 from hypothalamus stimulates LHX3 expression in the oral ectoderm, which is required for the maintenance of pituitary progenitor cells to prevent apoptosis. 
mice exhibited pituitary hypoplasia with enhanced apoptosis (28, $45)$. It has been previously reported that OTX2 binds to the promoters of HESX1 and POU1F1, which are expressed in the oral ectoderm and play an important role in its development, suggesting that oral ectodermal OTX2 may also be essential for its development (46). However, our results demonstrated that not oral ectodermal but hypothalamic OTX2 is essential for pituitary development, by regulating LHX3 expression in the oral ectoderm via FGF10 expression in the hypothalamus. Although LHX3 protein expression was observed in OTX2 ${ }^{\text {mut }}$-iPSCs (Figure $4 \mathrm{H}$ ) by addition of FGF10 into culture media, the oral ectoderm layer was still thin, and the LHX3 mRNA expression was not restored to a level comparable to that of control-iPSCs or OTX2 ${ }^{\text {mut }}$-repaired-iPSCs (Supplemental Figure 11). Interestingly, on addition of FGF8 accompanied by FGF10, the layer thickness was restored (Figure $4 \mathrm{H}$ and Supplemental Figure $7 \mathrm{E}$ ), although FGF8 solely did not restore the LHX3 expression (Figure 4, $\mathrm{G}$ and $\mathrm{H}$ ). These data suggested that FGF8 and FGF10 synergistically promote pituitary differentiation and proliferation of oral ectoderm, and inhibit apoptosis. However, long-term treatment with FGF8 and FGF10 could not induce pituitary hormone-producing cells (data not shown). It is speculated that in this protocol, although LHX3 was restored by FGF10, the expression levels were not enough for further differentiation as a possibility. Another possible explanation is that hedgehog signal, as well as FGF signaling, may play a role in the impaired differentiation of OTX $2^{\text {mut }}$-iPSCs. Although there were no significant differences, GLI1 and GLI2 expression, readouts of SHH signaling, tended to decrease in OTX2 ${ }^{\text {mut }}$-iPSCs (Supplemental Figure 6A). Because SHH also promotes LHX3 expression (37), downregulated $\mathrm{SHH}$ signaling might additionally impair induction into pituitary hormone-producing cells.

The precise mechanism by which OTX2 regulates FGF expression is still unclear. There have been several reports describing the interaction between OTX2 and FGF in neural tissues $(15,33,47)$. Both positive and negative correlation between OTX2 and FGF has been observed, suggesting that FGF may not be a direct target for OTX2. Interestingly, hypothalamus-specific Otx2-deficient mice exhibited impaired anterior pituitary development, and the importance of FGF signal from hypothalamus has been suggested (15), which was compatible with the present study. Our results with patient-derived and OTX2-knockout iPSCs demonstrated that OTX2 was required for the expression of FGF8 and FGF10 in the hypothalamus. It has been previously reported that during midbrain development, Otx2 regulates Fgf8 through the Limx1b and Wnt1 pathway (48). A similar pathway may also play a role in the hypothalamus. Alternatively, a secondary effect of structural abnormalities in the hypothalamus may also play a role.

This approach has several limitations. First, although the tissue develops to the hypothalamus and pituitary simultaneously in this method, it lacks a pituitary portal system that is essential for the appropriate traffic of hypothalamic hormones to the pituitary. These conditions may result in the incomplete differentiation of anterior pituitary cells. Second, the protocol requires several external growth factors and chemicals that are essential for pituitary differentiation, suggesting that these factors may mask developmental deficiencies in iPSCs from some patients. Also, the efficiency of the differentiation may vary depending on the cell lines. Therefore, it is important to interpret the results carefully, especially when the phenotype was subtle. In these aspects, animal models including knockout mice may be superior; therefore, it is recommended to apply these approaches complementarily. Third, the differentiation protocol still needs to be optimized, because the maturity of ACTH- and GH-producing cells and the differentiation of some lineages, such as TSH- and PRL-producing cells, are not enough. Although these limitations may restrict application for some conditions, we demonstrated the usefulness of this approach as a tool for human cell biology in pituitary development and disease.

In conclusion, we established a human in vitro model for $\mathrm{CPH}$ based on patient-derived iPSCs and showed at least a part of the underlying mechanisms by which the mutation in OTX2 caused $\mathrm{CPH}$. This method is a useful tool for studying human pituitary development and disease modeling and for elucidating the underlying mechanisms.

\section{Methods}

Endocrinological evaluation. Endocrinological evaluation of pituitary function was performed by the standard method (49). For the provocative tests, serum levels of ACTH, cortisol, TSH, GH, LH, FSH, and PRL were measured before injection and at 15, 30, 60, 90, and 120 minutes after i.v. administration of $0.25 \mathrm{U}$ insulin/ $\mathrm{kg}, 500 \mu \mathrm{g} \mathrm{TRH}$, and $100 \mathrm{~g} \mathrm{GnRH}$. Basal, peak, and reference values are described in Supplemental Table 1.

Generation and maintenance of iPSCs. For generation of patientderived iPSCs, OTX2, SOX2, KLF4, L-MYC, LIN28, and carboxyterminal dominant-negative fragment for $\mathrm{p} 53$ were transduced in peripheral blood leukocytes using episomal vectors as previously described $(25,50)$. Three healthy subject-derived iPSC lines (201B7, ref. 17; 409B2, ref. 50; and HC06 no. 4, ref. 51) were used in the study for control. Information on iPSCs used in this study is summarized in Supplemental Table 1. iPSCs were maintained on mitomycin C-treated SNL feeder cells (MSTO) in DMEM/F12 (Sigma-Aldrich) supplemented with 20\% (vol/vol) Knockout Serum Replacement (KSR; Thermo Fisher Scientific), $2 \mathrm{mM}$ L-glutamine (Thermo Fisher Scientific), 1× nonessential amino acid (Sigma-Aldrich), $80 \mu \mathrm{M}$ 2-mercaptoethanol (Sigma-Aldrich), and $7.5 \mathrm{ng} / \mathrm{mL}$ basic FGF (Wako).

Exome sequencing and data analysis. Next-generation sequencing using genomic DNA extracted from peripheral leukocytes was performed as previously described (52). We used the SureSelect All Exon kit (V2 or $50 \mathrm{Mb}$; Agilent Technologies) to capture whole exomes according to the manufacturer's protocol. The DNA obtained was subjected to paired-end sequencing with an Illumina sequencer (Genome Analyzer IIx or Hiseq; $2 \times 75 \mathrm{bp}$ and $2 \times 100 \mathrm{bp}$, respectively). Base calling, read filtering, and demultiplexing were performed with the standard Illumina processing pipeline. We used Burrows Wheeler Aligner (BWA) 0.7.5 to map reads against the human reference genome (NCBI Build 37; hg19) with default settings. Local realignment, quality score recalibration, and variant calling were performed with GATK 3.1-0 using the default settings. We used ANNOVAR for annotation of the called variants. With regard to genes associated with combined pituitary hormone deficiency (4), we only detected a heterozygous mutation in OTX2. The mutation was validated by PCR-directed sequencing.

In vitro induction of pituitary-hypothalamus from iPSCs. Pituitaryhypothalamus induction was done as previously described with some modifications (22). In brief, after removal of feeder cells using CTK 
solution ( $0.25 \%$ trypsin [Invitrogen], $0.1 \mathrm{mg} / \mathrm{mL}$ collagenase type IV [Invitrogen], $10 \mathrm{mM} \mathrm{CaCl}$ [Nacalai Tesque], and 20\% KSR in PBS), iPSCs were dissociated into single cells using Accutase (Nacalai Tesque). Then, 10,000 cells were plated in each well of a V-bottom low-attachment 96-well plate (Sumitomo Bakelite) in growth factor-free chemically defined medium (gfCDM) (53) supplemented with 10\% (vol/vol) of KSR. From day 0 to day 3, $20 \mu \mathrm{M}$ Y-27632 (Wako) and 100 nM LDN-193189 (Stemgent) were added. From day 6, 5 nM BMP4 (R\&D Systems) and 2 $\mu$ M SAG (Cayman Chemical) were added. From day 18, BMP4 was withdrawn and aggregates were maintained under the high- $\mathrm{O}_{2}$ condition (40\%). From day 30, aggregates were cultured in gfCDM supplemented with $20 \%$ (vol/vol) KSR. Induction into pituitary progenitor cells (LHX3+) was evaluated at day 40 . Induction into pituitary hormone-producing cells and ability to secrete these hormones were evaluated at day 100 . The FGF receptor inhibitor PD-173074 and recombinant human FGF8 and FGF10 were obtained from Millipore and R\&D Systems, respectively. Hypothalamus area, indicated by " $\mathrm{H}$ " in the figures, was defined as $\mathrm{RX}^{+}$ area in the aggregates. Oral ectoderm area, indicated by "O," was defined as E-cadherin ${ }^{+}$area outside of the aggregates.

Chimera culture experiment using GFP-labeled control-iPSCs. GFP-labeled iPSCs were generated with pHULK piggyBack Mammalian Expression Vector containing CometGFP and puromycin-resistant gene (ATUM). After selection with puromycin, a $\mathrm{GFP}^{+}$clone was used. For induction culture, 5000 cells of GFP-labeled control-iPSCs no. 1

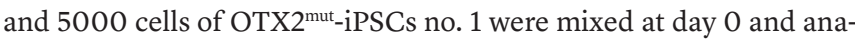
lyzed at day 40 .

To exclude the possibility that the GFP gene undergoes gene silencing in GFP-labeled iPSCs, we induced only from GFP-labeled control-iPSCs no. 1. Immunostaining revealed that all cells in the aggregates retained GFP expression, except for the necrotic area in the center of the aggregates, indicating that gene silencing did not occur (Supplemental Figure 5, B and C).

Plasmid construction for OTX 2 expression. OTX 2 cDNA was cloned from iPSC-derived hypothalamus-pituitary tissue. OTX2 isoform $b$ was expressed in iPSC-derived hypothalamus-pituitary. Then OTX2 cDNA was inserted into pc.DNA3.1D/V5-His (Thermo Fisher Scientific). The mutant OTX2 cDNA was created by site-directed mutagenesis using an overlapping PCR method. Wild-type and mutant OTX2 expression plasmid was transfected into HEK293T cells (obtained from ATCC) using X-tremeGENE HP DNA Transfection Reagent (Sigma-Aldrich) according to the manufacturer's instruction.

Immunostaining. Aggregates or cells were fixed with $4 \%$ paraformaldehyde for 15-30 minutes at room temperature. After overnight incubation in $20 \%$ sucrose, aggregates were embedded in OCT compound (Sakura Finetek) and frozen. Then, samples were cryosectioned at 8-10 $\mu \mathrm{m}$ thickness. After permeabilization with $0.3 \%$ Triton $\mathrm{X}-100$, slides were incubated with primary antibodies overnight at $4^{\circ} \mathrm{C}$, washed, and incubated with secondary antibody for 2 hours at room temperature. The primary antibodies used in this study are summarized in Supplemental Table 2. Secondary antibodies were species-specific Alexa Fluor conjugates (Thermo Fisher Scientific). Fluorescence images were obtained using a BZ-X700 microscope (Keyence) or LSM700 confocal microscopy (Zeiss).

Quantitative RT-PCR. Total RNA was extracted from 6-8 aggregates using an RNeasy Mini kit (Qiagen). For RNA extraction from a small number of FACS-sorted cells, TRIzol Reagent and Phasemaker Tubes (Thermo Fisher Scientific) were used. Total RNA was subjected to reverse transcription using ReverTra Ace qPCR RT Kit (TOYOBO). Quantitative PCR was performed with the Step One Plus Real-Time PCR system (Applied Biosystems) or LightCycler (Roche) using SYBR mix Ex Taq II (Takara). The thermal cycling profiles were as follows: initial denaturation at $95^{\circ} \mathrm{C}$ for 15 minutes, followed by 45 cycles of denaturation at $95^{\circ} \mathrm{C}$ for 15 seconds, annealing at $60^{\circ} \mathrm{C}$ for 30 seconds, and extension at $72^{\circ} \mathrm{C}$ for 15 seconds. $A C T B$ was used as an internal control. Each primer sequence used in the experiments is shown in Supplemental Table 3. All samples were assayed in duplicate.

Western blotting. Aggregates were washed with PBS and lysed in M-PER lysis buffer (Thermo Fisher Scientific) supplemented with cOmplete Protease Inhibitor Cocktail (Sigma-Aldrich). Fifteen micrograms of protein per lane was subjected to SDS-PAGE, and then transferred to a PVDF membrane. The membrane was incubated with primary antibody overnight at $4^{\circ} \mathrm{C}$. After incubation with HRP-conjugated secondary antibody for 1 hour at room temperature, signals were visualized with Immobilon Western Chemiluminescent HRP Substrate (Millipore). Images were obtained using an Amersham Imager 600 (GE Healthcare Life Science).

FACS. To separate cells derived from oral ectoderm and hypothalamic tissue, 50-100 aggregates were subjected to FACS. After washing of the aggregates twice with PBS, aggregates were dissociated into single cells treated with $5 \mathrm{mM}$ EDTA/HBSS for 10 minutes. Then, cells were filtered using a 100- $\mu \mathrm{m}$ cell strainer and incubated with Alexa Fluor-conjugated antibody against E-cadherin in FACS buffer (2\% FBS and $20 \mathrm{mM}$ HEPES in PBS) on ice for 30 minutes. After washing twice with FACS buffer, cells were resuspended in FACS buffer. Cells were filtered with a 100- $\mu \mathrm{m}$ cell strainer again and analyzed on a BD FACSAria III (BD Biosciences). E-cadherin-positive (defined as oral ectoderm tissue) and -negative (hypothalamus tissue) cells were sorted into culture medium and subjected to RNA extraction.

Sequencing analysis. Genomic DNA was extracted from peripheral blood and cultured cells using a NucleoSpin Tissue kit (MACHEREY-NAGEL). DNA sequence analysis of patient and iPSCs was performed by PCR-directed sequence as previously described (54). DNA sequence of cells with frameshift mutation (Figure 6A) was determined after cloning into the pCR2.1 vector with TA cloning (Thermo Fisher Scientific).

Analysis of ACTH and GH secretion. Six aggregates were cultured in $1 \mathrm{~mL}$ of gfCDM with or without $1 \mu \mathrm{g} / \mathrm{mL}$ CRH (Mitsubishi Tanabe Pharma) or GHRH (Sumitomo Dainippon Pharma). After 24 hours of incubation, culture medium was sampled, and concentration of ACTH and GH was determined by electrochemiluminescence immunoassay (ECLIA) using an Elecsys human ACTH kit and an Elecsys human GH kit (Roche Diagnostics), respectively.

CRISPR/Cas9 genome editing and screening of off-target mutation. Single guide RNA (sgRNA) sequences were designed using the Zhang laboratory CRISPR design tool (http://crispr.mit.edu; no longer available), and single-stranded oligodeoxynucleotide (ssODN) sequence was designed using the Edit-R HDR donor designer tool by Dharmacon (https://horizondiscovery.com/en/products/tools/EditR-HDR-Donor-Designer-oligo). Sequences of sgRNA and ssODN are listed in Supplemental Table 4. To improve the efficiency of homologous recombination, synonymous mutation was included in ssODN sequence as previously described (55). iPSCs were transfected with Cas9- and sgRNA-expressing plasmid (pX458 or pX459, Addgene plasmids 48138 and 62988) and ssODN using Lipofectamine Stem 
reagent (Thermo Fisher Scientific). After dissociation into single cells using Accutase, $3.75 \times 10^{5}$ cells were seeded with $2.5 \mu \mathrm{g}$ Cas9- and sgRNA-expressing plasmid, 50 pmol ssODN (PAGE-purified), and $10 \mu \mathrm{L}$ Lipofectamine Stem reagent in $2 \mathrm{~mL}$ culture medium supplemented with $10 \mu \mathrm{M}$ Y-27632. After selection with FACS (for pX458) or puromycin (for pX459), cells were transferred to MSTO-coated plates. Then, for limited dilution, 500 cells were seeded to $10-\mathrm{cm}$ plates in the presence of $10 \mu \mathrm{M}$ Y-27632. Desired homologous recombination was screened by restriction fragment length polymorphism (RFLP) with BmeT110I (Takara). RFLP $^{+}$clones were further analyzed with PCRdirected sequencing.

All mutation-repaired OTX2 ${ }^{\text {mut }}$-iPSC lines were tested for off-target editing events (56). The top 4 predicted off-target sites for the sgRNA sequence were listed using the CRISPOR Web tool (http:// crispor.tefor.net/). The region including each off-target site was PCR-amplified and sequenced. Predicted sites and primer sequences are listed in Supplemental Table 5.

Statistics. Data are expressed as means \pm SEM or box-and-whisker plots. Continuous data were compared by a Student's $t$ test or Wilcoxon rank-sum test. Multiple groups were compared by a 1-way ANOVA or Kruskal-Wallis test with a post hoc multiple comparison. Two-tailed $P$ values of 0.05 or less were considered statistically significant. Statistical analysis was performed using R (version 3.2.4) (57).

Study approval. All experimental protocols dealing with human subjects were approved by the Ethics Committee of Kobe University Graduate School of Medicine (approval number 1646). Written informed consent was obtained from the patient.

\section{Author contributions}

RM, HS, TA, and YT designed the studies. RM performed the in vitro experiments. HF and GI provided analyzed samples. SN and TH performed genetic analysis. KM established iPSCs. RM, HS, $\mathrm{HB}, \mathrm{HF}, \mathrm{GI}, \mathrm{WO}, \mathrm{TA}$, and YT interpreted the data. RM and YT drafted the manuscript.

\section{Acknowledgments}

We are grateful to Y. Oiso at Nagoya University and T. Yamamoto at Kyoto University for their support, N. Matsumoto at Yokohama City University for exome sequencing analysis, and the members of the Takahashi, Suga, and Aoi laboratories for valuable discussions. This work was supported by grants from the Japan Agency for Medical Research and Development (AMED; The Acceleration Program for Intractable Disease Research Utilizing Disease-specific iPS Cells: JP 18bm0804012h0002), grants-in-aid for Scientific Research from the Ministry of Education, Culture, Sports, Science and Technology (17K19684 and 16H05332 to YT and 18K16232 to $\mathrm{RM})$, grants from the Uehara Memorial Foundation and the Naito Foundation, and the Program for Intractable Disease Research Utilizing Disease-specific iPS Cells of the Japan Science and Technology Agency/AMED (16bm0609002h0105 to KM).

Address correspondence to: Yutaka Takahashi, Department of Diabetes and Endocrinology, Kobe University Graduate School of Medicine, 7-5-1, Kusunoki-cho, Chuo-ku, Kobe, Hyogo 650-0017, Japan. Phone: 81.78.382.5861; Email: takahash@med.kobe-u.ac.jp.
1. Castinetti F, et al. Mechanisms in endocrinology: an update in the genetic aetiologies of combined pituitary hormone deficiency. Eur JEndocrinol. 2016;174(6):R239-R247.

2. Cohen LE, Radovick S. Molecular basis of combined pituitary hormone deficiencies. Endocr Rev. 2002;23(4):431-442.

3. Prince KL, Walvoord EC, Rhodes SJ. The role of homeodomain transcription factors in heritable pituitary disease. Nat Rev Endocrinol. 2011;7(12):727-737.

4. Fang $Q$, et al. Genetics of combined pituitary hormone deficiency: roadmap into the genome era. Endocr Rev. 2016;37(6):636-675.

5. Kelberman D, Rizzoti K, Lovell-Badge R, Robinson IC, Dattani MT. Genetic regulation of pituitary gland development in human and mouse. Endocr Rev. 2009;30(7):790-829.

6. Alatzoglou KS, Dattani MT. Genetic forms of hypopituitarism and their manifestation in the neonatal period. Early Hum Dev. 2009;85(11):705-712.

7. Chatelain G, Fossat N, Brun G, Lamonerie T. Molecular dissection reveals decreased activity and not dominant negative effect in human OTX2 mutants. JMol Med. 2006;84(7):604-615.

8. Acampora D, et al. Forebrain and midbrain regions are deleted in Otx2-/- mutants due to a defective anterior neuroectoderm specification during gastrulation. Development. 1995;121(10):3279-3290.

9. Elliott J, Maltby EL, Reynolds B. A case of deletion $14(\mathrm{q} 22.1 \rightarrow \mathrm{q} 22.3)$ associated with anophthalmia and pituitary abnormalities. JMed Genet.
1993;30(3):251-252

10. Tajima T, Ishizu K, Nakamura A. Molecular and clinical findings in patients with LHX4 and OTX2 mutations. Clin Pediatr Endocrinol. 2013;22(2):15-23.

11. Dateki S, et al. Heterozygous orthodenticle homeobox 2 mutations are associated with variable pituitary phenotype. J Clin Endocrinol Metab. 2010;95(2):756-764.

12. Diaczok D, Romero C, Zunich J, Marshall I, Radovick S. A novel dominant negative mutation of OTX2 associated with combined pituitary hormone deficiency. J Clin Endocrinol Metab. 2008;93(11):4351-4359.

13. Ferran JL, Puelles L, Rubenstein JL. Molecular codes defining rostrocaudal domains in the embryonic mouse hypothalamus. Front Neuroanat. 2015;9:46.

14. Spieler D, et al. Involvement of Pax6 and Otx2 in the forebrain-specific regulation of the vertebrate homeobox gene ANF/Hesx1. Dev Biol. 2004;269(2):567-579.

15. Mortensen AH, Schade V, Lamonerie T, Camper SA. Deletion of OTX2 in neural ectoderm delays anterior pituitary development. Hum Mol Genet. 2015;24(4):939-953.

16. Mortensen AH, MacDonald JW, Ghosh D, Camper SA. Candidate genes for panhypopituitarism identified by gene expression profiling. Physiol Genomics. 2011;43(19):1105-1116.

17. Takahashi $\mathrm{K}$, et al. Induction of pluripotent stem cells from adult human fibroblasts by defined factors. Cell. 2007;131(5):861-872.

18. Avior Y, Sagi I, Benvenisty N. Pluripotent stem cells in disease modelling and drug discovery. Nat Rev Mol Cell Biol. 2016;17(3):170-182.

19. Dincer Z, et al. Specification of functional cranial placode derivatives from human pluripotent stem cells. Cell Rep. 2013;5(5):1387-1402.

20. Zimmer B, Piao J, Ramnarine K, Tomishima MJ, Tabar V, Studer L. Derivation of diverse hormone-releasing pituitary cells from human pluripotent stem cells. Stem Cell Reports. 2016;6(6):858-872.

21. Suga $\mathrm{H}$, et al. Self-formation of functional adenohypophysis in three-dimensional culture. Nature. 2011;480(7375):57-62.

22. Ozone $\mathrm{C}$, et al. Functional anterior pituitary generated in self-organizing culture of human embryonic stem cells. Nat Commun. 2016;7:10351.

23. Sasai Y, Eiraku M, Suga H. In vitro organogenesis in three dimensions: self-organising stem cells. Development. 2012;139(22):4111-4121.

24. Gorbenko Del Blanco D, Romero CJ, Diaczok D, de Graaff LC, Radovick S, Hokken-Koelega AC. A novel OTX2 mutation in a patient with combined pituitary hormone deficiency, pituitary malformation, and an underdeveloped left optic nerve. Eur JEndocrinol. 2012;167(3):441-452.

25. Okita K, et al. An efficient nonviral method to generate integration-free human-induced pluripotent stem cells from cord blood and peripheral blood cells. Stem Cells. 2013;31(3):458-466.

26. Pfaeffle RW, et al. Four novel mutations of the LHX3 gene cause combined pituitary hormone deficiencies with or without limited neck rotation. JClin Endocrinol Metab. 2007;92(5):1909-1919.

27. De Rienzo F, et al. Frequency of genetic defects in 
combined pituitary hormone deficiency: a systematic review and analysis of a multicentre Italian cohort. Clin Endocrinol (Oxf). 2015;83(6):849-860.

28. Ellsworth BS, Butts DL, Camper SA. Mechanisms underlying pituitary hypoplasia and failed cell specification in Lhx3-deficient mice. Dev Biol. 2008;313(1):118-129.

29. Inoue F, Kurokawa D, Takahashi M, Aizawa S. Gbx2 directly restricts Otx2 expression to forebrain and midbrain, competing with class III POU factors. Mol Cell Biol. 2012;32(13):2618-2627.

30. Muranishi Y, et al. An essential role for RAX homeoprotein and NOTCH-HES signaling in Otx2 expression in embryonic retinal photoreceptor cell fate determination. J Neurosci. 2011;31(46):16792-16807.

31. Schilter KF, et al. OTX2 microphthalmia syndrome: four novel mutations and delineation of a phenotype. Clin Genet. 2011;79(2):158-168.

32. Zhu X, Gleiberman AS, Rosenfeld MG. Molecular physiology of pituitary development: signaling and transcriptional networks. Physiol Rev. 2007;87(3):933-963.

33. Broccoli V, Boncinelli E, Wurst W. The caudal limit of Otx2 expression positions the isthmic organizer. Nature. 1999;401(6749):164-168.

34. Sakurai Y, Kurokawa D, Kiyonari H, Kajikawa E, Suda Y, Aizawa S. Otx2 and Otx1 protect diencephalon and mesencephalon from caudalization into metencephalon during early brain regionalization. Dev Biol. 2010;347(2):392-403.

35. Greber B, et al. FGF signalling inhibits neural induction in human embryonic stem cells. $E M B O J$. 2011;30(24):4874-4884.

36. Carreno G, et al. Hypothalamic sonic hedgehog is required for cell specification and proliferation of LHX3/LHX4 pituitary embryonic precursors. Development. 2017;144(18):3289-3302.

37. Treier M, et al. Hedgehog signaling is required for pituitary gland development. Development. 2001;128(3):377-386.
38. McCabe MJ, Dattani MT. Genetic aspects of hypothalamic and pituitary gland development. Handb Clin Neurol. 2014;124:3-15.

39. Zwaveling-Soonawala N, et al. Clues for polygenic inheritance of pituitary stalk interruption syndrome from exome sequencing in 20 patients. JClin Endocrinol Metab. 2018;103(2):415-428.

40. Guo QH, et al. Multi-genic pattern found in rare type of hypopituitarism: a whole-exome sequencing study of Han Chinese with pituitary stalk interruption syndrome. J Cell Mol Med. 2017;21(12):3626-3632.

41. Maione L, et al. Genetics in endocrinology: Genetic counseling for congenital hypogonadotropic hypogonadism and Kallmann syndrome: new challenges in the era of oligogenism and next-generation sequencing. Eur J Endocrinol. 2018;178(3):R55-R80.

42. Ericson J, Norlin S, Jessell TM, Edlund T. Integrated FGF and BMP signaling controls the progression of progenitor cell differentiation and the emergence of pattern in the embryonic anterior pituitary. Development. 1998;125(6):1005-1015.

43. Ohuchi H, et al. FGF10 acts as a major ligand for FGF receptor 2 IIIb in mouse multi-organ development. Biochem Biophys Res Commun. 2000;277(3):643-649.

44. De Moerlooze L, Spencer-Dene B, Revest JM, Hajihosseini M, Rosewell I, Dickson C. An important role for the IIIb isoform of fibroblast growth factor receptor 2 (FGFR2) in mesenchymal-epithelial signalling during mouse organogenesis. Development. 2000;127(3):483-492.

45. Zhao Y, Morales DC, Hermesz E, Lee WK, Pfaff SL, Westphal H. Reduced expression of the LIM-homeobox gene Lhx3 impairs growth and differentiation of Rathke's pouch and increases cell apoptosis during mouse pituitary development. Mech Dev. 2006;123(8):605-613.

46. Dateki S, Fukami M, Sato N, Muroya K, Adachi $\mathrm{M}$, Ogata T. OTX2 mutation in a patient with anophthalmia, short stature, and partial growth hormone deficiency: functional studies using the IRBP, HESX1, and POU1F1 promoters. J Clin Endocrinol Metab. 2008;93(10):3697-3702.

47. Acampora D, Postiglione MP, Avantaggiato V, Di Bonito M, Simeone A. The role of Otx and Otp genes in brain development. Int J Dev Biol. 2000;44(6):669-677.

48. Harada H, Sato T, Nakamura H. Fgf8 signaling for development of the midbrain and hindbrain. Dev Growth Differ. 2016;58(5):437-445.

49. Vance ML. Hypopituitarism. N EnglJMed. 1994;330(23):1651-1662.

50. Okita K, et al. A more efficient method to generate integration-free human iPS cells. Nat Methods. 2011;8(5):409-412.

51. Ishida $Y$, et al. Vulnerability of Purkinje cells generated from spinocerebellar ataxia type 6 patientderived iPSCs. Cell Rep. 2016;17(6):1482-1490.

52. Narumi S, et al. SAMD9 mutations cause a novel multisystem disorder, MIRAGE syndrome, and are associated with loss of chromosome 7. Nat Genet. 2016;48(7):792-797.

53. Wataya T, et al. Minimization of exogenous signals in ES cell culture induces rostral hypothalamic differentiation. Proc Natl Acad Sci U S A. 2008;105(33):11796-11801.

54. Matsumoto R, et al. Genetic and clinical characteristics of Japanese patients with sporadic somatotropinoma. Endocr J. 2016;63(11):953-963.

55. Paquet D, et al. Efficient introduction of specific homozygous and heterozygous mutations using CRISPR/Cas9. Nature. 2016;533(7601):125-129.

56. Haeussler M, et al. Evaluation of off-target and on-target scoring algorithms and integration into the guide RNA selection tool CRISPOR. Genome Biol. 2016;17(1):148.

57. R Core Team. R: A Language and Environment for Statistical Computing. R Foundation for Statistical Computing. Vienna, Austria. https://www.Rproject.org/. Accessed November 13, 2019. 